\title{
The Price of Anarchy for Selfish Ring Routing is Two
}

XUJIN CHEN, Institute of Applied Mathematics, AMSS, Chinese Academy of Sciences, China BENJAMIN DOERR, Max Planck Institute for Informatics, Germany, and LIX, École Polytechnique, France CAROLA DOERR, Max Planck Institute for Informatics, Germany, and LIAFA, Université Paris Diderot - Paris 7, France

XIAODONG HU, Institute of Applied Mathematics, AMSS, Chinese Academy of Sciences, China WEIDONG MA, Institute of Applied Mathematics, AMSS, Chinese Academy of Sciences, China ROB VAN STEE, Max Planck Institute for Informatics, Germany

We analyze the network congestion game with atomic players, asymmetric strategies, and the maximum latency among all players as social cost. This important social cost function is much less understood than the average latency. We show that the price of anarchy is at most two, when the network is a ring and the link latencies are linear. Our bound is tight. This is the first sharp bound for the maximum latency objective.

Categories and Subject Descriptors: C.2.1 [Computer-Communication Networks]: Network Architecture and Design-Network communications; C.2.2 [Computer-Communication Networks]: Network Protocols-Routing protocols

General Terms: Theory, Performance

Additional Key Words and Phrases: Price of anarchy, selfish routing, congestion games

ACM Reference Format:

Xujin Chen, Benjamin Doerr, Carola Doerr, Xiaodong Hu, Weidong Ma, and Rob van Stee, 2013. The Price of Anarchy for Selfish Ring Routing is Two. ACM Trans. Econ. Comp. V, N, Article A ( 2013), 23 pages.

DOI :http://dx.doi.org/10.1145/0000000.0000000

\section{INTRODUCTION}

Selfish routing is a fundamental problem in algorithmic game theory, and was one of the first problems which were intensively studied in this field [Koutsoupias and Papadimitriou 2009; Mavronicolas and Spirakis 2001; Roughgarden and Tardos 2002; Czumaj 2004]. A main question in this field concerns the cost of selfishness: how much performance is lost because agents behave selfishly, without regard for the other agents or for any global objective function?

The established measure for this performance loss is the price of anarchy (PoA) [Koutsoupias and Papadimitriou 2009]. This is the worst-case ratio between the value of a Nash equilibrium, where no player can deviate unilaterally to improve, and the value of the optimal routing.

A preliminary version of this article appeared in the WINE'12 conference proceedings.

Author's addresses: X. Chen, X. Hu and W. Ma, Institute of Applied Mathematics, AMSS, Chinese Academy of Sciences, Beijing 100190, China; B. Doerr and C. Doerr, Max Planck Institute for Informatics, Campus E1 4, 66123 Saarbrücken, Germany; R. van Stee, Department of Computer Science, University of Leicester, United Kingdom.

Permission to make digital or hard copies of part or all of this work for personal or classroom use is granted without fee provided that copies are not made or distributed for profit or commercial advantage and that copies show this notice on the first page or initial screen of a display along with the full citation. Copyrights for components of this work owned by others than ACM must be honored. Abstracting with credit is permitted. To copy otherwise, to republish, to post on servers, to redistribute to lists, or to use any component of this work in other works requires prior specific permission and/or a fee. Permissions may be requested from Publications Dept., ACM, Inc., 2 Penn Plaza, Suite 701, New York, NY 10121-0701 USA, fax +1 (212) 869-0481, or permissions@acm.org.

(c) 2013 ACM 1946-6227/2013/-ARTA $\$ 15.00$

DOI : http://dx.doi.org/10.1145/0000000.0000000 
Of particular interest to computer science are network congestion games, where agents choose routing paths and experience delays (latencies) depending on how much other players also use the edges on their paths. Such games are guaranteed to admit at least one Nash equilibrium [Rosenthal 1973]. Generally, the PoA for a selfish routing problem may depend on the network topology, the number of players (including the non-atomic case where an infinite number of players each controls a negligible fraction of the solution), the type of latency functions on the links, and the objective functions of the players and of the system (the latter is often called the social cost function).

Most of the existing research has focused on the PoA for minimizing the total latency of all the players [Roughgarden 2003; Aland et al. 2006]. Indeed, this measure is so standard that it is often not even mentioned in titles or abstracts. In most cases, a symmetric setting was considered where all players have the same source node and the same destination node, and hence the same strategy set. Christodoulou and Koutsoupias [2005] and Awerbuch et al. [2005] independently proved that the PoA of the atomic congestion game (symmetric or asymmetric) with linear latency is at most 2.5. This bound is tight. The bound grows to 2.618 for weighted demands [Awerbuch et al. 2005], which is again a tight bound. In non-atomic congestion games with linear latencies, the PoA is at most 4/3 [Roughgarden and Tardos 2002]. This is witnessed already by two parallel links. The same paper also obtained a generalized result for polynomial latencies.

In this work, we regard as social cost function the maximum latency a player experiences. While this cost function was suggested already in [Koutsoupias and Papadimitriou 1999], it seems much less understood. For general topologies, the maximum PoA of atomic congestion games with linear latency is 2.5 in single-commodity networks (symmetric case, all player choose paths between the same pair of nodes), but it grows to $\Theta(\sqrt{k})$ in $k$-commodity networks (asymmetric case, $k$ players have different nodes to connect via a path) [Christodoulou and Koutsoupias 2005]. The PoA further increases with additional restrictions to the strategy sets. Gairing et al. [2006] showed that when the graph consists of $n$ parallel links and each player's choice can be restricted to a particular subset of these links, the maximum PoA lies in the interval $[n-1, n)$.

For non-atomic selfish routing, Lin et al. [2011] showed that the PoA of symmetric games on $n$-node networks with arbitrary continuous and non-decreasing latency functions is $n-1$, and exhibited an infinite family of asymmetric games whose PoA grows exponentially with the network size.

Our setting:: In this work, we analyze the price of anarchy of a maximum latency network congestion game for a concrete and useful network topology, namely rings. Rings are frequently encountered in communication networks. Seven self-healing rings form the EuroRings network, the largest, fastest, best-connected high-speed network in Europe, spanning 25,000 km and connecting 60 cities in 18 countries.

As its name suggests, the Global Ring Network for Advanced Applications Development [GLORIAD 2013] is an advanced science internet network constructed as an optical ring around the Northern Hemisphere. The global ring topology of the network provides scientists, educators and students with advanced networking tools, and enables active, daily collaboration on common problems.

It is therefore worthwhile to study this topology in particular. Indeed, considerable research has already gone into studying rings, in particular in the context of designing approximation algorithms for combinatorial optimization problems [Anshelevich and Zhang 2008; Blum et al. 2001; Cheng 2004; Schrijver et al. 1998; Wang 2005].

As in most previous work, we assume that traffic may not be split, because this causes the problem of packet reassembly at the receiver and is therefore generally avoided. Furthermore, we assume that the edges ("links") have linear latency func- 
tions. That is, each link $e$ has a latency function $\ell_{e}(x)=a_{e} x+b_{e}$, where $x$ is the number of players using link $e$ and $a_{e}$ and $b_{e}$ are nonnegative constants.

For the problem of minimizing the maximum latency on a ring, even assuming a central authority, the question of how to route communication requests optimally is nontrivial; to the best of our knowledge, it is not known whether this problem is in $\mathcal{P}$. It is known for general (directed or undirected) network topologies that already the price of stability $(\mathrm{PoS})$, which is the ratio of the value of the best Nash equilibrium to that of the optimal solution [Anshelevich et al. 2004], is unbounded for this objective function even for linear latency functions [Chen et al. 2010; 2013]. However, this is not the case for rings. It has been shown that for any instance on a ring, either its PoS equals 1 , or its PoA is at most 6.83, giving a universal upper bound 6.83 on PoS for the selfish ring routing [Chen et al. 2010]. The same paper also gave a lower bound of 2 on the PoA. Recently, an upper bound of 16 on the PoA was obtained [Chen et al. 2013].

Our results: In this paper, we show that the PoA for minimizing the maximum latency on rings is exactly 2 . This improves upon the previous best known upper bounds on both the PoA and the PoS [Chen et al. 2013; 2010]. Achieving the tight bound required us to upper bound a high-dimensional nonlinear optimization problem. Our result implies that the performance loss due to selfishness is relatively low for this problem. Thus, for ring routing, simply allowing each agent to choose its own path will always result in reasonable performance.

Additional Remarks. The lower bound example (see Figure 1) can be modified to give a lower bound of $2^{d}$ for latency functions that are polynomials of degree at most $d$, cf. Section 8. Furthermore, we show that, for a generalization of this problem which we will call complement games, the PoA is at most 2.15.

Proof overview: Our proof consists of two main parts: first, we analyze for Nash equilibria the maximum ratio of the latency of any player to the latency of the entire ring, and then we analyze the ratio of the latency of the entire ring in a Nash equilibrium to the maximum player latency in an optimal routing. In the first part we show that this ratio is at most roughly $2 / 3$; the precise value depends on whether or not every link of the ring is used by at least one player in the Nash equilibrium.

For the second ratio, we begin by showing the very helpful fact that it is sufficient to consider only instances where no player uses the same path in the Nash routing as in the optimal routing. For such instances, we need to distinguish two cases. The first case deals with instances for which there exists a link that in the Nash equilibrium is not used by any player. For such instances we use a structural analysis to bound the second ratio from above by $2+2 / k$, where $k$ is the number of agents in the system.

For the main case in which the paths of the players in the Nash equilibrium cover the ring, we show that the second ratio is at most 3 . We begin by using the standard technique of adding up the Nash inequalities which state that no player can improve by deviating to its alternative path. This gives us a constraint which must be satisfied for any Nash equilibrium, but this does not immediately give us an upper bound for the second ratio. Instead, we end up with a nonlinear optimization problem: maximize the ratio under consideration subject to the Nash constraint. The analysis of this problem was the main technical challenge of this paper. We use a series of modifications to reach an optimization problem with only five variables, which, however, is still nonlinear. It can be solved by Maple, but we also provide a formal solution.

\section{THE SELFISH RING ROUTING MODEL}

Let $\mathcal{I}=\left(\mathcal{R}, \ell,\left(s_{i}, t_{i}\right)_{i \in[k]}\right)$ be a selfish ring routing $(\mathrm{SRR})$ instance, where $\mathcal{R}=(V, E)$ is a ring and where for each agent $i \in[k]$ the pair $\left(s_{i}, t_{i}\right)$ denotes the source and the 
destination nodes of agent $i$. We sometimes refer to the agents as players. For every link $e \in E$ we denote the latency function by $\ell_{e}(x)=a_{e} x+b_{e}$, where $a_{e}$ and $b_{e}$ are nonnegative constants; without loss of generality we assume that $a_{e}, b_{e}$ are nonnegative integers. This is feasible since real-valued inputs can be approximated arbitrarily well by integers by scaling the input appropriately.

For any subgraph $\mathcal{P}$ of $\mathcal{R}$ (written as $\mathcal{P} \subseteq \mathcal{R}$ ), we slightly abuse the notation and identify $\mathcal{P}$ with its link set $E(\mathcal{P})$. If $\mathcal{Q}$ is a path on $\mathcal{R}$ with end nodes $s$ and $t$, we use $\mathcal{P} \backslash \mathcal{Q}$ to denote the graph obtained from $\mathcal{P}$ by removing all nodes in $V(\mathcal{P}) \cap V(\mathcal{Q}) \backslash\{s, t\}$ (all internal nodes of $\mathcal{Q}$ which are contained in $\mathcal{P}$ ), and all links in $\mathcal{P} \cap \mathcal{Q}$ (all links of $\mathcal{Q}$ which are contained in $\mathcal{P}$ ).

For any feasible routing $\pi=\left\{\mathcal{P}_{1}, \ldots, \mathcal{P}_{k}\right\}$, where $\mathcal{P}_{i}$ is a path on $\mathcal{R}$ between $s_{i}$ and $t_{i}$, $i=1, \ldots, k$, we denote by $M(\pi):=\max _{i \in[k]} \ell\left(\mathcal{P}_{i}, \pi\right)$ the maximum latency of any of the $k$ agents. Here we abbreviate by $\ell(\mathcal{P}, \pi)$ the latency

$$
\ell(\mathcal{P}, \pi):=\sum_{e \in \mathcal{P}}\left(a_{e}\left|\left\{i \in[k] \mid e \in \mathcal{P}_{i}\right\}\right|+b_{e}\right)
$$

of a subgraph $\mathcal{P} \subseteq \mathcal{R}$ in $\pi$. We say that $\pi$ is a Nash equilibrium (routing) if no agent $i \in[k]$ can reduce its latency $\ell\left(\mathcal{P}_{i}, \pi\right)$ by switching $\mathcal{P}_{i}$ to the alternative path $\mathcal{R} \backslash \mathcal{P}_{i}$, provided other agents do not change their paths.

Sometimes we are only interested in the latency caused by one additional agent and we write $\|\mathcal{P}\|_{a}:=\sum_{e \in \mathcal{P}} a_{e}$. Similarly we abbreviate $\|\mathcal{P}\|_{b}:=\sum_{e \in \mathcal{P}} b_{e}$.

Let $\pi^{N}=\left\{\mathcal{N}_{1}, \ldots, \mathcal{N}_{k}\right\}$ be some fixed worst Nash routing (i.e., a Nash equilibrium with maximum system latency $M\left(\pi^{N}\right)$ ), and let $\Pi^{*}$ be the set of optimal routings of $\mathcal{I}$.

For any $\pi=\left\{\mathcal{Q}_{1}, \ldots, \mathcal{Q}_{k}\right\} \in \Pi^{*}$, let

$$
h(\pi):=\left|\left\{i \in[k]: \mathcal{N}_{i} \neq \mathcal{Q}_{i}\right\}\right| .
$$

I.e., $h(\pi)$ is the number of agents for which their Nash routings are not the same as their optimal routings. We choose $\pi^{*}=\left\{\mathcal{Q}_{1}, \ldots, \mathcal{Q}_{k}\right\} \in \Pi^{*}$ to be an optimal routing that minimizes $h=h\left(\pi^{*}\right)$. Without loss of generality, we assume that $\left\{i \in[k]: \mathcal{N}_{i} \neq \mathcal{Q}_{i}\right\}=$ $[h]:=\{1, \ldots, h\}$. We call the agents $1, \ldots, h$ switching agents and we refer to the agents in $[k] \backslash[h]$ as non-switching ones.

For brevity, we write $P^{*}:=\ell\left(\mathcal{P}, \pi^{*}\right)$ and $P:=\ell\left(\mathcal{P}, \pi^{N}\right)$. Similarly, we let $M:=M\left(\pi^{N}\right)$ and $M^{*}:=M\left(\pi^{*}\right)$. Abusing notation, for any link $e \in \mathcal{R}$, we set

$$
\pi^{*}(e):=\left|\left\{i \in[h] \mid e \in \mathcal{Q}_{i}\right\}\right|,
$$

the number of switching players whose optimal paths traverse $e$. Analogously, $\pi^{N}(e):=$ $\left|\left\{i \in[h] \mid e \in \mathcal{N}_{i}\right\}\right|$.

\section{MAIN RESULT AND OUTLINE OF THE PROOF}

The purpose of this paper is the proof of the following statement.

THEOREM 3.1. The price of anarchy for selfish ring routing with linear latencies is 2 .

As mentioned in the introduction, a simple example for which the price of anarchy is two has been given already in [Chen et al. 2010]. This is the example depicted in Figure 1 . As is easy to verify, $M^{*}=1$ and $M=2$. Hence, our result is tight. It suffices to prove the upper bound in Theorem 3.1. That is, we need to show that for all SRR instances $\mathcal{I}$ the ratio $M / M^{*}$ is at most two.

\subsection{Main Steps}

The main steps for proving $M / M^{*} \leq 2$ are as follows. 

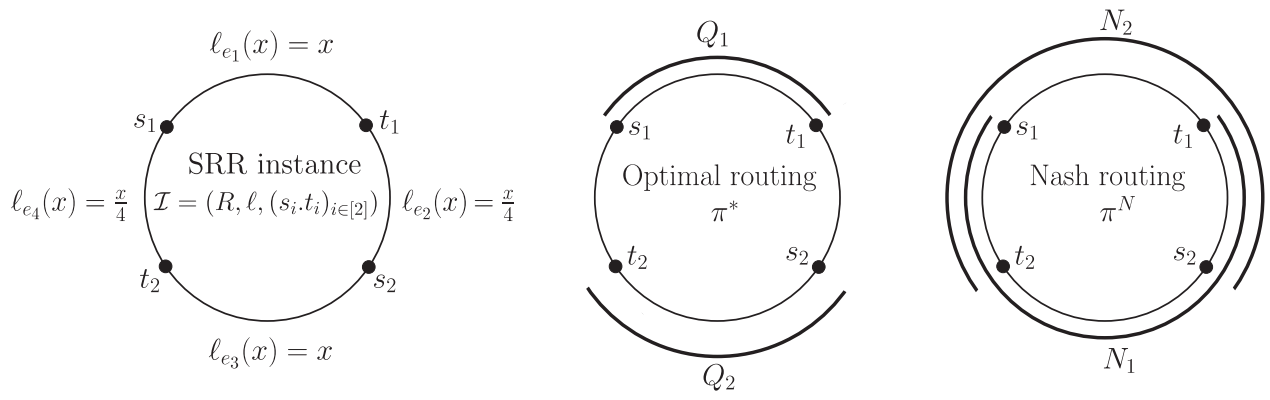

Fig. 1. A 2-player SRR instance with $\mathrm{PoA}=2$.

1. We begin by restricting the set of Nash routings we need to consider. We show that we can assume without loss of generality that in $\pi^{N}$ there is at most one player that uses the same path as in $\pi^{*}$, i.e., $h \geq k-1$ (Section 3.2). We call the case where there is such a player the singular case; if there is not such a player, we are in the nonsingular case.

2. We say that the Nash equilibrium $\pi^{N}$ is a covering equilibrium if the Nash paths of the switching agents $1, \ldots, h$ cover the ring, i.e., if $\cup_{i \in[h]} \mathcal{N}_{i}=\mathcal{R}$. For any noncovering equilibrium, we use a structural analysis of $\pi^{N}$ to show (Section 4 ) that the PoA is less than two for $h \geq 3$.

3 . We proceed by showing (Lemma 5.1) that for every covering equilibrium, the ratio $M / R$ is at most $2 / 3$, where $R=\ell\left(\mathcal{R}, \pi^{N}\right)$ is the total latency of all links on $R$ under the Nash routing $\pi^{N}$.

4. Finally, in the remainder of Section 5 , we show that $R / M^{*} \leq 3$ for any covering equilibrium $\pi^{N}$. This is the main part of the proof. Combining this with the third statement concludes the proof of Theorem 3.1 for covering equilibria.

Some specific cases with small values of $h$ need to be handled separately. Our proof needs the following technical lemma which is true for both covering and non-covering equilibria. It shows that any two Nash paths of agents that use different paths in $\pi^{N}$ and in $\pi^{*}$ share at least one common link.

LEMMA 3.2. For all $i, j \in[h]$, the optimal paths $\mathcal{Q}_{i}$ and $\mathcal{Q}_{j}$ do not jointly cover the entire ring, equivalently their complements $\mathcal{N}_{i}$ and $\mathcal{N}_{j}$ are not link-disjoint.

Proof. Assume there exist two agents $i, j \in[h]$ such that $\mathcal{Q}_{i} \cup \mathcal{Q}_{j}=\mathcal{R}$. Consider the routing $\pi^{\prime}$ which is exactly the same as $\pi^{*}$, except for these two agents $i$ and $j$. For any link $e \in \mathcal{Q}_{i} \cap \mathcal{Q}_{j}$ we have $\pi^{\prime}(e)=\pi^{*}(e)-2$, and for every link $e \in\left(\mathcal{Q}_{i} \backslash \mathcal{Q}_{j}\right) \cup\left(\mathcal{Q}_{j} \backslash \mathcal{Q}_{i}\right)$ the number of agents on this link does not change, i.e., $\pi^{\prime}(e)=\pi^{*}(e)$. Since $a_{e} \geq 0$ for all $e \in E$, this yields $M\left(\pi^{\prime}\right) \leq M^{*}$. Hence, $\pi^{\prime} \in \Pi^{*}$. But we also have $h\left(\pi^{\prime}\right)<h\left(\pi^{*}\right)$, contradicting the choice of $\pi^{*}$ given in Section 2.

\subsection{Reduction to Singular and Nonsingular Instances}

The next lemma shows that we only need consider the case where at most one player is not switching.

LEMMA 3.3. Consider any selfish ring routing instance $\mathcal{I}=\left(\mathcal{R}, \ell,\left(s_{i}, t_{i}\right)_{i \in[k]}\right)$ with linear latencies. Let $\pi^{*}$ be an optimal routing and let $\pi^{N}$ be a Nash routing. Suppose there is an agent $q \in[k]$ that uses the same path in $\pi^{N}$ as in $\pi^{*}$. Then there exists a selfish routing instance $\mathcal{I}^{\prime}=\left(\mathcal{R}, \ell^{\prime},\left(s_{i}, t_{i}\right)_{i \in[k] \backslash\{q\}}\right)$ with linear latency functions $\ell_{e}^{\prime}(x)$ such that 
- the non-switching agent $q$ is removed from $\mathcal{I}$ to get $\mathcal{I}^{\prime}$,

- the routing $\pi^{N}$ restricted to the remaining agents, denoted as $\pi^{N^{\prime}}$, is a Nash equilibrium for $\mathcal{I}^{\prime}$,

- the total ring latencies satisfy $\ell^{\prime N}(\mathcal{R}):=\ell^{\prime}\left(\mathcal{R}, \pi^{N^{\prime}}\right)=\ell^{N}(\mathcal{R})$, and

- we have $M^{\prime}\left(\mathrm{opt}^{\prime}\right) \leq M\left(\pi^{*}\right)$ for the maximum latencies of individual agents. Here, $\mathrm{opt}^{\prime}$ denotes an optimal routing for $\mathcal{I}^{\prime}$ and $M^{\prime}(\cdot)$ denotes the maximum latency of a routing in $\mathcal{I}^{\prime}$.

Proof. By definition, player $q$ uses path $\mathcal{Q}_{q}$ in both $\pi^{N}=\left\{\mathcal{N}_{i}: i \in[k]\right\}$ and $\pi^{*}=$ $\left\{\mathcal{Q}_{i}: i \in[k]\right\}$. Remove player $q$ from $\mathcal{I}$. For every link $e \in \mathcal{Q}_{q}$ set $\ell_{e}^{\prime}(x):=\ell_{e}(x)+$ $a_{e}=a_{e} x+b_{e}+a_{e}$. The latency functions of all other links are unchanged. Denote the resulting instance $\left(\mathcal{R}, \ell^{\prime},\left(s_{i}, t_{i}\right)_{i \in[k] \backslash\{q\}}\right)$ by $\mathcal{I}^{\prime}$.

Every routing $\pi$ for $\mathcal{I}$ induces a routing $\pi^{\prime}$ for $\mathcal{I}^{\prime}$ in the natural way, by omitting the routing for player $q$. From the modified latency defined above, we see that the latency of every edge in an induced routing is the same as the original latency in $\mathcal{I}$. It follows immediately that

- a routing which is a Nash equilibrium in $\mathcal{I}$ induces a Nash equilibrium routing in $\mathcal{I}^{\prime}$

- the latency of the entire ring of an induced routing is also the same as the ring latency of the original routing in $\mathcal{I}$, and

- the maximum latency of the induced routing $\pi^{* \prime}$ of the optimal routing $\pi^{*}$ is not larger than the maximum latency of the optimal routing itself, i.e., $M^{\prime}\left(\pi^{* \prime}\right) \leq M\left(\pi^{*}\right)$.

By definition, the optimal routing opt ${ }^{\prime}$ for instance $\mathcal{I}^{\prime}$ cannot be worse than the feasible routing $\pi^{* \prime}$, and we conclude $M^{\prime}\left(\mathrm{opt}^{\prime}\right) \leq M^{\prime}\left(\pi^{* \prime}\right) \leq M\left(\pi^{*}\right)$.

We call the Nash routing $\pi^{N}$ singular if $M\left(\pi^{N}\right)>\max _{i \in[h]} \ell^{N}\left(\mathcal{N}_{i}\right)$, i.e., if the maximum latency in $\pi^{N}$ is obtained only by an agent which uses the same routing in $\pi^{N}$ as it uses in $\pi^{*}$. We call $\pi^{N}$ nonsingular otherwise. That is, $\pi^{N}$ is nonsingular if $M=M\left(\pi^{N}\right)=\max _{i \in[h]} \ell^{N}\left(\mathcal{N}_{i}\right)$. Since we are interested in upper bounding the ratio $M / M^{*}$, applying Lemma 3.3 repeatedly enables us to make the following assumption.

ASSUMPTION 1. $h \leq k \leq h+1$ and $h=k-1$ if and only if $\pi^{N}$ is singular.

Under Assumption 1, for any singular case $\left(\pi^{N}, \mathcal{I}\right)$, Lemma 3.3 produces a nonsingular case $\left(\pi^{N^{\prime}}, \mathcal{I}^{\prime}\right)$ with $\ell^{\prime N}\left(\mathcal{R}, \mathcal{I}^{\prime}\right) / M^{\prime}\left(o p t^{\prime}, \mathcal{I}^{\prime}\right) \geq \ell^{N}(\mathcal{R}, \mathcal{I}) / M\left(\pi^{*}, \mathcal{I}\right)$. Therefore we can upper bound the price of anarchy, which is the worst-case ratio $M / M^{*}$, for the SRR problem as follows:

- upper bound the ratio $R / M^{*}=\ell^{N}(\mathcal{R}, \mathcal{I}) / M\left(\pi^{*}, \mathcal{I}\right)$ only for nonsingular instances $\mathcal{I}$ where no player uses the same path in $\pi^{N}$ and $\pi^{*}$, and

- upper bound the ratio $M / R=M\left(\pi^{N}, \mathcal{I}\right) / \ell^{N}(\mathcal{R}, \mathcal{I})$ for general instances $\mathcal{I}$.

This is what we will do in the remainder of the paper.

\section{NON-COVERING EQUILIBRIA}

In this section, we use a structural analysis on the non-covering equilibrium $\pi^{N}$ to show that the PoA is less than two for $h \geq 3$.

THEOREM 4.1. The ratio $M / M^{*}$ is at most $\frac{4}{3}+\frac{5}{3 h}$ for instances with $\cup_{i \in[h]} \mathcal{N}_{i} \neq \mathcal{R}$.

The proof of Theorem 4.1 consists of the following two steps. First we show that the ratio $R / M^{*}$ is at most $2+\frac{2}{h}$. This is Lemma 4.2. Next we show (Lemma 4.3) that for any uncovered instance, if $R / M^{*} \leq \alpha$ for some constant $\alpha$, then $M / M^{*}$ is at most 


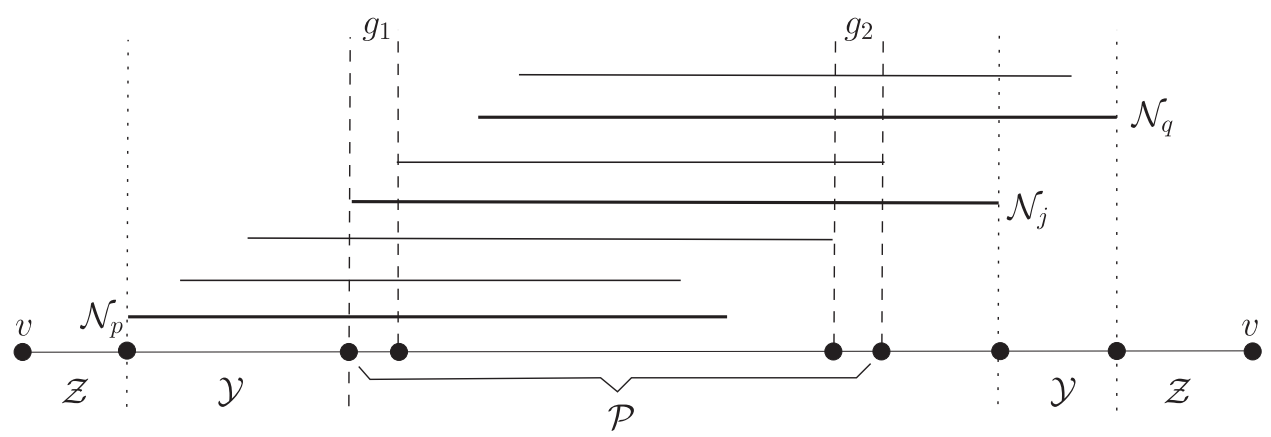

Fig. 2. Proof for non-covering equilibria. For this figure, we have mapped the ring to the real interval shown at the bottom whose both ends represent the same node $v$ of the ring $\mathcal{R}$.

$\left(2 \alpha+\frac{1}{h}\right) / 3$. This proves Theorem 4.1, which itself proves Theorem 3.1 for the noncovered case with $h \geq 3$. The remaining case of non-covering equilibria with $h=2$ is handled in Section 6 , where we show $M / M^{*} \leq 2$ directly by utilizing the structural properties of rings.

LEMmA 4.2. Let $\mathcal{I}$ be an SRR instance with $\cup_{i \in[h]} \mathcal{N}_{i} \neq R$. Then $R / M^{*} \leq 2+\frac{2}{h}$.

Proof. By Lemma 3.3, it suffices to consider the nonsingular case. That is, we assume without loss of generality that $k=h$, i.e., we assume that $\mathcal{N}_{j}=\mathcal{R}-\mathcal{Q}_{j}$ for all $j$.

Consider an arbitrary link $e$ that is not used in any Nash path. Order the players by nondecreasing distance (in links) of their clockwise first endpoint from $e$. We claim that all $h$ paths in $\mathcal{N}_{1}, \mathcal{N}_{2}, \ldots, \mathcal{N}_{h}$ share a common link. This holds because if there were three agents $i_{1}<i_{2}<i_{3}$ (using the ordering above) that do not all share a same link, then $i_{1}$ and $i_{3}$ would not share a link at all, since $\cup_{i \in[h]} \mathcal{N}_{i} \neq \mathcal{R}$. However, this contradicts Lemma 3.2.

Let $p$ be an agent with an endpoint closest to $e$ in the clockwise direction, and $q$ be an agent with an endpoint closest to $e$ in the counterclockwise direction. Then by the above $\cup_{i \in[h]} \mathcal{N}_{i} \subseteq \mathcal{N}_{p} \cup \mathcal{N}_{q} \varsubsetneqq \mathcal{R}$. Let $\mathcal{P}$ be the longest path in $\mathcal{N}_{p} \cup \mathcal{N}_{q}$ with end links $g_{1}$ and $g_{2}$ (possibly $\left\{g_{1}\right\}=\left\{g_{2}\right\}=\mathcal{P}$ ) such that $\pi^{N}\left(g_{i}\right)>h / 2$ for $i=1,2$ and

$$
\pi^{N}(g) \leq h / 2 \text { for any link } g \in \mathcal{N}_{p} \cup \mathcal{N}_{q} \backslash \mathcal{P} \text {. }
$$

See Figure 2. Since we have $g_{1}=g_{2}$ or $\pi^{N}\left(g_{1}\right)+\pi^{N}\left(g_{2}\right)>h$, there exists an agent $j \in[h]$ such that $\left\{g_{1}, g_{2}\right\} \subseteq \mathcal{N}_{j}$ and thus $\mathcal{P} \subseteq \mathcal{N}_{j}$. Let $\mathcal{Y} \subseteq \mathcal{Q}_{j}$ consist of links $e$ with $\pi^{N}(e) \geq 1$ and $\mathcal{Z}=\mathcal{Q}_{j} \backslash \mathcal{Y}$. It can be seen from (1) that $Q_{j} \leq \frac{h}{2}\|\mathcal{Y}\|_{a}+\|\mathcal{Y}\|_{b}+\|\mathcal{Z}\|_{b}$ and therefore

$$
\begin{aligned}
R & =Q_{j}+N_{j} \leq 2 Q_{j}+\|\mathcal{Y}\|_{a}+\|\mathcal{Z}\|_{a} \quad \text { (by definition of a Nash equilibrium) } \\
& \leq(h+1)\|\mathcal{Y}\|_{a}+2\|\mathcal{Y}\|_{b}+\|\mathcal{Z}\|_{a}+2\|\mathcal{Z}\|_{b} .
\end{aligned}
$$

Since

$$
Q_{j}^{*} \geq \frac{h}{2}\|\mathcal{Y}\|_{a}+\|\mathcal{Y}\|_{b}+h\|\mathcal{Z}\|_{a}+\|\mathcal{Z}\|_{b},
$$

the ratio of the upper bound (2) for $R$ to the lower bound (3) for $Q_{j}^{*}$ is maximized for $\|\mathcal{Z}\|_{a}=\|\mathcal{Z}\|_{b}=\|\mathcal{Y}\|_{b}=0$ and is $(h+1) /(h / 2)=2+2 / h$.

To conclude the proof of Theorem 4.1, we finally show the following.

LEMmA 4.3. The ratio $M / M^{*}$ is at most $\left(2 \alpha+\frac{1}{h}\right) / 3$ for instances with $R / M^{*} \leq \alpha$. 
PROOF. It suffices to show that for any agent $i \in[k]$ the inequality $N_{i} \leq \frac{1}{3}\left(2 \alpha+\frac{1}{h}\right) M^{*}$ holds. Consider an arbitrary agent $i \in[k]$. Let $\mathcal{C}_{i}:=\mathcal{R} \backslash \mathcal{N}_{i}$, the complement of player $i$ 's path $\mathcal{N}_{i}$. We partition the link set of $\mathcal{C}_{i}$ into the set of links $\mathcal{Y}:=\left\{e \in \mathcal{C}_{i} \mid \pi^{N}(e) \geq 1\right\}$ which, in routing $\pi^{N}$, have at least one agent on it and the set of links $\mathcal{Z}:=\mathcal{C}_{i} \backslash \mathcal{Y}$ with no players on it in routing $\pi^{N}$. (The set $\mathcal{Z}$ might be empty.)

Since $h$ is the number of players whose paths in $\pi^{N}$ deviate from the one in $\pi^{*}$, the links $e$ in $\mathcal{Z}$ satisfy $\pi^{*}(e) \geq h$, that is, there are at least $h$ players using these links in the routing $\pi^{*}$. Hence $M^{*} \geq h\|\mathcal{Z}\|_{a}$. In the routing $\pi^{N}$, if player $i$ would switch from path $\mathcal{N}_{i}$ to $\mathcal{C}_{i}$, it would have a latency of at most $C_{i}+\|\mathcal{Y}\|_{a}+\|\mathcal{Z}\|_{a}$. Since $\pi^{N}$ is a Nash equilibrium, we have $N_{i} \leq C_{i}+\|\mathcal{Y}\|_{a}+\|\mathcal{Z}\|_{a} \leq 2 C_{i}+\frac{1}{h} M^{*}$ and

$$
3 N_{i} \leq 2\left(C_{i}+N_{i}\right)+\frac{1}{h} M^{*}=2 R+\frac{1}{h} M^{*}
$$

By assumption we also have $R \leq \alpha M^{*}$. Thus (4) gives $3 N_{i} \leq\left(2 \alpha+\frac{1}{h}\right) M^{*}$, as required.

\section{COVERING EQUILIBRIA}

For covering equilibria, we show that the price of anarchy is at most 2 . This is again a two-step approach. First, the covering property implies an upper bound of $2 / 3$ on $M / R$ as follows.

LEMMA 5.1. If $\cup_{i \in[h]} \mathcal{N}_{i}=\mathcal{R}$, then $M / R \leq 2 / 3$.

Proof. Take $\mathcal{N} \in \pi^{N}$ with $N=M$. Then $N \leq \ell^{N}(\mathcal{R} \backslash \mathcal{N})+\|\mathcal{R} \backslash \mathcal{N}\|_{a}$, and therefore $R=N+\ell^{N}(\mathcal{R} \backslash \mathcal{N}) \geq 2 N-\|\mathcal{R} \backslash \mathcal{N}\|_{a} \geq 2 N-\ell^{N}(\mathcal{R} \backslash \mathcal{N})=3 N-R$, where the second inequality holds because $\pi^{N}$ is covering. We deduce that $M=N \leq \frac{2}{3} R$.

Second, we prove $R / M^{*} \leq 3$ by distinguishing between the case $h \leq 2$ and $h>2$.

THEOREM 5.2. If $\cup_{i \in[h]} \mathcal{N}_{i}=\mathcal{R}$, then $R / M^{*} \leq 3$.

The former case $h \leq 2$ is proved in Section 6, which along with Lemma 5.14 in this section establishes Theorem 5.2.

By Lemma 3.3, we only need to bound ratio $R / M^{*}$ for nonsingular case where $h=k$. In this section we consider the $k=h \geq 3$ switching players. For each switching player $i \in[h]$, we can formulate an inequality $N_{i} \leq Q_{i}+\left\|\mathcal{Q}_{i}\right\|_{a}$ saying that its Nash path may not have a longer latency than its alternative path, if one unit load is added on every link of the latter. We obtain a constraint by adding up all of these inequalities.

We can assume that every link has a latency function of $x$ or 1 . This can be achieved by replacing a link $e$ with latency function $a_{e} x+b_{e}$ by $a_{e}$ links with latency function $x$ followed by $b_{e}$ links with latency function 1 . Now there are only two types of links left, the ones with latency function $x$ and the ones with latency 1 . We introduce variables which count the number of links of both types which are used by a certain number of players, and write the constraint that we constructed above in terms of these variables. We then give an upper bound for $R / M^{*}$ in terms of these variables as well.

We end up with a nonlinear optimization problem: maximize the ratio under consideration subject to the Nash constraint. For this problem, we first show that, for the links with latency function 1, roughly speaking, only the total number of players on all these links affects the upper bound. By normalization, for any fixed number of players $h$ that do not use the same path in the Nash routing as in the optimal routing, this still leaves us with $h+2$ variables, since we have one variable for each possible number of players on the links with latency function $x$, and two other variables (see (8)-(11)).

We now use a centering argument to show that only at most two of the $h$ player variables are nonzero in an optimal solution of this optimization problem. This finally 
gives us an optimization problem with five variables, where $h$ is also considered a variable (see (15)-(19)). This problem unfortunately is still not linear. It can be solved by Maple, but we also provide a formal solution. To do this, we fix $h$ and another variable, and solve the remaining problem; we then determine the optimal overall values of the fixed $h$ and that variable.

Summing the Nash inequalities. For a given path $\mathcal{P} \subseteq \mathcal{R}$, let $\mathcal{P}^{a}$ be the subset of links with latency function $x$ and let $\mathcal{P}^{b}$ be the subset of links with latency function 1.

Consider a link $e \in \mathcal{R}^{a}$ (resp. $\mathcal{R}^{b}$ ). By definition and our assumption that $k=h$, this link occurs in $\pi^{N}(e)$ Nash paths. That is, this link occurs $\pi^{N}(e)$ times on the left-hand side of the $h$ Nash inequalities given above-each time with coefficient $\pi^{N}(e)$ (resp. 1). On the other hand, it occurs $h-\pi^{N}(e)$ times on the right-hand side of the inequalities, each time with coefficient $\pi^{N}(e)+1$ (resp. 1).

Formally, we have for $i=1, \ldots, h$

$$
\sum_{e \in \mathcal{N}_{i}^{a}} \pi^{N}(e)+\sum_{e \in \mathcal{N}_{i}^{b}} 1=N_{i} \leq Q_{i}+\left\|\mathcal{Q}_{i}\right\|_{a}=\sum_{e \in \mathcal{Q}_{i}^{a}}\left(\pi^{N}(e)+1\right)+\sum_{e \in \mathcal{Q}_{i}^{b}} 1
$$

and, by summation,

$$
\begin{aligned}
& \sum_{e \in R^{a}}\left(\pi^{N}(e)\right)^{2}+\sum_{e \in R^{b}} \pi^{N}(e) \leq \sum_{e \in R^{a}}\left(h-\pi^{N}(e)\right)\left(\pi^{N}(e)+1\right)+\sum_{e \in R^{b}}\left(h-\pi^{N}(e)\right), \\
& \text { or } \sum_{e \in R^{a}}\left(2\left(\pi^{N}(e)\right)^{2}-h\right)+\sum_{e \in R^{b}} 2 \pi^{N}(e) \leq \sum_{e \in R^{a}}(h-1) \pi^{N}(e)+\sum_{e \in R^{b}} h .
\end{aligned}
$$

Writing $A_{i}$ (resp. $B_{i}$ ) as the number of links with $i$ players on it in $\pi^{N}$ and a latency function of $x$ (resp. 1), we can group links with the same numbers of players and write the above as

$$
\begin{aligned}
\sum_{i=1}^{h}\left(\left(2 i^{2}-h\right) A_{i}+2 i B_{i}\right) & \leq \sum_{i=1}^{h}\left((h-1) i A_{i}+h B_{i}\right) \\
\Rightarrow \sum_{i=1}^{h}\left(\left(\frac{2 i}{h}-\frac{1}{i}\right) C_{i}+\frac{2 i}{h^{2}} B_{i}\right) & \leq \sum_{i=1}^{h}\left(\frac{h-1}{h} C_{i}+\frac{1}{h} B_{i}\right)
\end{aligned}
$$

where we have written $C_{i}=\frac{i}{h} A_{i}$ and divided by $h^{2}$.

Bounding the optimal latency. For the optimal routing we also have, by definition and the fact that we are in the nonsingular case, $h$ inequalities of the form $M^{*} \geq \ell^{*}\left(\mathcal{Q}_{i}\right)$, $i \in[h]$. Summing all the inequalities and dividing by $h$ implies a lower bound on $M^{*}$, namely

$$
M^{*} \geq \frac{1}{h} \sum_{i=1}^{h} \ell^{*}\left(\mathcal{Q}_{i}\right)=\frac{1}{h} \sum_{i=1}^{h}\left((h-i)^{2} A_{i}+(h-i) B_{i}\right) .
$$

Note that $A_{i}$ and $B_{i}$ still have the same meanings as before, and refer to $\pi^{N}$, not $\pi^{*}$. Thus we have

$$
\frac{R}{M^{*}} \leq \frac{\sum_{i=1}^{h}\left(i A_{i}+B_{i}\right)}{\sum_{i=1}^{h}\left(\frac{(h-i)^{2}}{h} A_{i}+\frac{h-i}{h} B_{i}\right)}=\frac{\sum_{i=1}^{h}\left(C_{i}+\frac{1}{h} B_{i}\right)}{\sum_{i=1}^{h}\left(\frac{(h-i)^{2}}{i h} C_{i}+\frac{h-i}{h^{2}} B_{i}\right)}
$$

and we want to find an upper bound for this expression under the restriction (6).

LEMMA 5.3. If $\sum_{i=1}^{h} C_{i}=0$, then $R / M^{*} \leq 2$. 
Proof. Since $C_{i} \geq 0$ by definition, we have $C_{i}=0$ for all $i \in[h]$. Condition (6) implies that $\sum_{i=1}^{h} \frac{i}{h} B_{i} \leq \frac{1}{2} \sum_{i=1}^{h} B_{i}$. Therefore, by (7), the ratio $R / M^{*}$ is at most $\left(\sum_{i=1}^{h} B_{i}\right) /\left(\sum_{i=1}^{h} B_{i}-\sum_{i=1}^{h} \frac{i}{h} B_{i}\right) \leq\left(\sum_{i=1}^{h} B_{i}\right) /\left(\frac{1}{2} \sum_{i=1}^{h} B_{i}\right)=2$.

Rewriting the problem. Henceforth we assume $\sum_{i=1}^{h} C_{i}>0$. Using $\frac{(h-i)^{2}}{i h}=\frac{h}{i}+$ $\frac{i}{h}-2$, from (7) we arrive at the following inequality after dividing numerator and denominator by $\sum_{j=1}^{h} C_{j}>0$.

$$
\begin{aligned}
\frac{R}{M^{*}} & \leq \frac{1+\sum_{i=1}^{h} \frac{B_{i}}{h \sum_{j=1}^{h} C_{j}}}{\sum_{i=1}^{h}\left(\left(\frac{h}{i}+\frac{i}{h}\right) \frac{C_{i}}{\sum_{j=1}^{h} C_{j}}+\frac{h-i}{h^{2}} \frac{B_{i}}{\sum_{j=1}^{h} C_{j}}\right)-2} \\
& \leq \frac{1+\beta}{\sum_{i=1}^{h}\left(\frac{h}{i}+\frac{i}{h}\right) D_{i}-2+\beta-z}
\end{aligned}
$$

where $\beta:=\frac{\sum_{i=1}^{h} B_{i}}{h \sum_{j=1}^{h} C_{j}} \geq 0, z:=\sum_{i=1}^{h} \frac{i B_{i}}{h^{2} \sum_{j=1}^{h} C_{j}} \in\left[\frac{\beta}{h}, \beta\right]$, and $D_{i}:=\frac{C_{i}}{\sum_{j=1}^{h} C_{j}}$ for every $i \in[h]$. Notice that $\sum_{i=1}^{h} D_{i}=1$. We divide both sides of (6) by $\sum_{j=1}^{h} C_{j}$ and obtain the constraint $\sum_{i=1}^{h}\left(\frac{2 i}{h}-\frac{1}{i}\right) D_{i}+2 z \leq \frac{h-1}{h}+\beta$. Our problem now looks as follows.

$$
\begin{aligned}
& \frac{R}{M^{*}} \leq \max \frac{1+\beta}{\sum_{i=1}^{h}\left(\frac{h}{i}+\frac{i}{h}\right) D_{i}-2+\beta-z} \\
& \text { s.t. } \sum_{i=1}^{h}\left(\frac{2 i}{h}-\frac{1}{i}\right) D_{i}+2 z \leq \frac{h-1}{h}+\beta \\
& \sum_{i=1}^{h} D_{i}=1, \quad D_{i} \geq 0 \forall i \in[h] \\
& \beta / h \leq z \leq \beta
\end{aligned}
$$

To bound the ratio $R / M^{*}$ from above we will solve the general problem (8)-(11), where we ignore our definitions of $\beta$ and $z$ above and thus allow $\beta$ and $z$ to take any nonnegative real values (subject to (11)).

Since $\frac{h}{i}+\frac{i}{h} \geq 2$ for all $i \geq 1$ and $h \geq 1$, we see that for any $\beta \geq 0$ and $h \geq 1$, the denominator in (8) is positive for every feasible solution $\left(\left\{D_{i}\right\}_{i=1}^{h}, z\right)$ of (9)-(11). We can therefore also consider the following equivalent minimization problem:

$$
\min \left\{\sum_{i=1}^{h}\left(\frac{h}{i}+\frac{i}{h}\right) D_{i}-z \mid(9)-(11)\right\} .
$$

In what follows, we solve (12) for any fixed $h$ and $\beta$, and then determine which values of $h$ and $\beta$ give the highest overall value for (8). For fixed $h$ and $\beta$, any solution $\left(\left\{D_{i}\right\}_{i=1}^{h}, z\right)$ of $(9)-(11)$ is either an optimal solution to both problem (8)-(11) and problem (12) or to neither of them. The next lemma helps to simplify our problem (12), and hence problem (8)-(11).

LEMMA 5.4. There is an optimal solution $\left(\left\{D_{i}\right\}_{i=1}^{h}, z\right)$ of (12), which is also an optimal solution of (8) - (11), such that $D_{i}>0$ for at most two values of $i$. If there are two such values, they are consecutive. 
Proof. Consider an optimal solution $\left(\left\{D_{i}\right\}_{i=1}^{h}, z\right)$ of (12). Suppose for a contradiction that there exist two indices $i_{1} \leq i_{2}-2$ such that $D_{i_{1}}>0$ and $D_{i_{2}}>0$. We can modify the solution $\left\{D_{i}\right\}_{i=1}^{h}$ as follows. Let $m_{j}(j=1,2)$ be real values with $0<m_{j} \leq D_{i_{j}}$. Subtract $m_{1}$ from $D_{i_{1}}$ and add it to $D_{i_{1}+1}$. Subtract $m_{2}$ from $D_{i_{2}}$ and add it to $D_{i_{2}-1}$. Then we still have $\sum_{i=1}^{h} D_{i}=1$ and $D_{i} \geq 0(i=1, \ldots, h)$.

We need to determine $m_{1}$ and $m_{2}$ so that constraint (9) is still satisfied. To this end, we investigate by how much the sum on the left-hand side of (9) increases, i.e.,

$$
\begin{aligned}
& \left(-\frac{2 i_{1}}{h}+\frac{1}{i_{1}}+\frac{2\left(i_{1}+1\right)}{h}-\frac{1}{i_{1}+1}\right) m_{1}+\left(\frac{2\left(i_{2}-1\right)}{h}-\frac{1}{i_{2}-1}-\frac{2 i_{2}}{h}+\frac{1}{i_{2}}\right) m_{2} \\
= & \left(\frac{1}{i_{1}}+\frac{2}{h}-\frac{1}{i_{1}+1}\right) m_{1}-\left(\frac{2}{h}+\frac{1}{i_{2}-1}-\frac{1}{i_{2}}\right) m_{2},
\end{aligned}
$$

which should be at most 0 in order to maintain a feasible solution. This is equivalent to requiring that $\frac{m_{1}}{m_{2}}$ be bounded from above by

$$
\left(\frac{1}{\left(i_{2}-1\right) i_{2}}+\frac{2}{h}\right) /\left(\frac{1}{\left(i_{1}+1\right) i_{1}}+\frac{2}{h}\right)=: \alpha_{i_{1} i_{2}} \leq 1,
$$

where the last inequality holds since $i_{2} \geq i_{1}+2$. On the other hand, we aim at decreasing the objective function in (12) with this procedure. Therefore, we require the increase of the objective value to be negative. From this we get

$$
\begin{aligned}
0 & >\left(-\frac{h}{i_{1}}-\frac{i_{1}}{h}+\frac{h}{i_{1}+1}+\frac{i_{1}+1}{h}\right) m_{1}+\left(\frac{h}{i_{2}-1}+\frac{i_{2}-1}{h}-\frac{h}{i_{2}}-\frac{i_{2}}{h}\right) m_{2} \\
& =\left(\frac{-h}{i_{1}\left(i_{1}+1\right)}+\frac{1}{h}\right) m_{1}+\left(\frac{h}{\left(i_{2}-1\right) i_{2}}-\frac{1}{h}\right) m_{2} \\
& \Rightarrow\left(\frac{h}{\left(i_{2}-1\right) i_{2}}-\frac{1}{h}\right) m_{2}<\left(\frac{h}{i_{1}\left(i_{1}+1\right)}-\frac{1}{h}\right) m_{1} .
\end{aligned}
$$

Note that the coefficients of $m_{1}$ and $m_{2}$ are positive since $i_{1}+2 \leq i_{2} \leq h$. Therefore, requiring that the increase of the objective function be negative is equivalent to requiring that $\frac{m_{1}}{m_{2}}$ be greater than

$$
\left(\frac{h}{\left(i_{2}-1\right) i_{2}}-\frac{1}{h}\right) /\left(\frac{h}{i_{1}\left(i_{1}+1\right)}-\frac{1}{h}\right)=: \beta_{i_{1} i_{2}}
$$

From the definitions in (13) and (14), doing crosswise multiplication, it is easy to check that $\beta_{i_{1} i_{2}}<\alpha_{i_{1} i_{2}}$ for $i_{1}+2 \leq i_{2} \leq h$. This shows that there exist positive values $m_{1}$ and $m_{2}$ such that $\alpha_{i_{1} i_{2}} \geq \frac{m_{1}}{m_{2}}>\beta_{i_{1} i_{2}}$, and $m_{j} \leq D_{i_{j}}$ for $j=1,2$. Thus, as a result of our modification of the sequence $\left\{D_{i}\right\}_{i=1}^{h}$, the objective function value in (12) decreases by a positive amount, and the constraints (9)-(11) are still satisfied. This contradicts the optimality of $\left(\left\{D_{i}\right\}_{i=1}^{h}, z\right)$.

For an optimal solution $\left(\left\{D_{i}\right\}_{i=1}^{h}, z\right)$ to (8)-(11) as given in Lemma 5.4, let $x \in[h-1]$ be the minimum index such that $D_{i}=0$ for all $i \in[h] \backslash\{x, x+1\}$. That is, $x$ is the minimum index such that $D_{x}>0$, or $x=h-1$. Writing $y$ for $D_{x+1}$, we have $D_{x}=1-y$, and problem (8) - (11) transforms to the following relaxation which drops the upper bound $\beta$ on $z$ in (11). 


$$
\begin{aligned}
\frac{R}{M^{*}} \leq \max & \frac{1+\beta}{\frac{h}{x}+\frac{x}{h}-\left(\frac{h}{x(x+1)}-\frac{1}{h}\right) y-2+\beta-z} \\
\text { s.t. } & \frac{2 x}{h}-\frac{1}{x}+\left(\frac{2}{h}+\frac{1}{x(x+1)}\right) y+2 z \leq \frac{h-1}{h}+\beta \\
& 1 \leq x \leq h-1, x \in \mathbb{N} \\
& 0 \leq y \leq 1 \\
& \beta / h \leq z
\end{aligned}
$$

For convenience, we restate the problem (15) - (19) as follows

$$
\frac{R}{M^{*}} \leq \max \left\{\frac{1+\beta}{f(x, y, z)} \mid g(x, y, z) \leq 0, x \in \mathbb{N}, y \in[0,1], z \geq \frac{\beta}{h}\right\},
$$

where we have relaxed the constraint on $x$ and

$$
\begin{aligned}
& f(x, y, z)=\frac{h}{x}+\frac{x}{h}-\left(\frac{h}{x(x+1)}-\frac{1}{h}\right) y-z-2+\beta, \\
& g(x, y, z)=\frac{2 x}{h}-\frac{1}{x}+\left(\frac{2}{h}+\frac{1}{x(x+1)}\right) y+2 z-\frac{h-1}{h}-\beta .
\end{aligned}
$$

We turn to consider the corresponding minimization of $f(x, y, z)$ under the same constraints. It is clear that the optimal value of the minimization is attained at $g(x, y, z)=0$ (if $g(x, y, z)<0$, we can increase $z$ and decrease the objective function). So we only need to consider the minimization problem with this equality constraint, as well as its relaxation, as follows:

$$
\begin{aligned}
& \Omega_{1}:=\min \{f(x, y, z) \mid g(x, y, z)=0, x \in \mathbb{N}, y \in[0,1], z \geq \beta / h\} \\
& \Omega_{2}:=\min \{f(x, y, z) \mid g(x, y, z)=0, x \geq 1, y \in[0,1], z \geq \beta / h\}
\end{aligned}
$$

OBSERVATION 1. For $i=1$ or 2 , if $\Omega_{i} \geq \frac{1+\beta}{3}$, then $\frac{R}{M^{*}} \leq 3$.

Main ideas. In view of Observation 1 , we will prove $\Omega_{1} \geq \frac{1+\beta}{3}$ for $h \in\{3,4,6\}$ in Section 5.1, and $\Omega_{2} \geq \frac{1+\beta}{3}$ for $h \geq 7$ in Section 5.2. The proof for $\Omega_{1}$ utilizes a case analysis, which is simplified by the fact that every optimal solution of (21) when $h \in$ $\{3,4,6\}$ has its $y$ or $z$ touch the boundary. The key idea for lower bounding $\Omega_{2}$ is using the fact that the optimal solution of (22) must be a KKT point (a solution satisfying the Karush-Kuhn-Tucker (KKT) conditions). We will bound the values of objective function $f$ at all KKT points of (22) from below by $\frac{1+\beta}{3}$.

To lower bound $\Omega_{1}$ and $\Omega_{2}$, we need to consider the derivatives of the objective and constraint functions. Using $x \geq 1$ and $y \in[0,1]$, we obtain

$$
\begin{array}{llll}
\frac{\partial f}{\partial x}=\frac{1}{h}-\frac{h(1-y)}{x^{2}}-\frac{h y}{(x+1)^{2}}, & \frac{\partial f}{\partial y}=\frac{1}{h}-\frac{h}{x(x+1)}, & & \frac{\partial f}{\partial z}=-1, \\
\frac{\partial g}{\partial x}=\frac{2}{h}+\frac{1-y}{x^{2}}+\frac{y}{(x+1)^{2}}, & \frac{\partial g}{\partial y}=\frac{2}{h}+\frac{1}{x(x+1)}, & \frac{\partial g}{\partial z}=2 .
\end{array}
$$

For brevity we define $\chi:=\left(\sqrt{2 h^{2}-h+1}-1\right) / 2$ and $\nu:=\sqrt{2 h^{2}-h} / 2$. It is straightforward to verify the following equivalences.

LEMMA 5.5 .

(i) $\frac{\partial f / \partial x}{\partial g / \partial x}=\frac{\partial f / \partial y}{\partial g / \partial y} \Leftrightarrow y=\frac{x+1}{2 x+1} \Leftrightarrow \frac{\partial f}{\partial x}=\frac{\partial f}{\partial y} \Leftrightarrow \frac{\partial g}{\partial x}=\frac{\partial g}{\partial y}$. 
(ii) $\frac{\partial f / \partial y}{\partial g / \partial y} \geq \frac{\partial f / \partial z}{\partial g / \partial z} \Leftrightarrow x \geq \chi$, and $\frac{\partial f / \partial y}{\partial g / \partial y}=\frac{\partial f / \partial z}{\partial g / \partial z} \Leftrightarrow x=\chi$.

(iii) If $y \in\{0,1\}$, then $\frac{\partial f / \partial x}{\partial g / \partial x}=\frac{\partial f / \partial z}{\partial g / \partial z} \Leftrightarrow x+y=\nu$.

(iv) If $y=\frac{x+1}{2 x+1}$, then $\frac{\partial f / \partial x}{\partial g / \partial x}=\frac{\partial f / \partial z}{\partial g / \partial z} \Leftrightarrow x=\chi$.

LEMMA 5.6. Let $\left(x^{*}, y^{*}, z^{*}\right)$ be an optimal solution to (21) or (22).

(i) If $x^{*}<\chi$, then $y^{*}=1$ or $z^{*}=\beta / h$.

(ii) If $x^{*}>\chi$, then $y^{*}=0$.

(iii) $x^{*} \leq h-1$.

PROOF. (i)-(ii) If $x^{*}<\chi$ (resp. $x^{*}>\chi$ ), then it follows from Lemma 5.5(ii) that $\frac{\partial f / \partial y}{\partial g / \partial y}<\frac{\partial f / \partial z}{\partial g / \partial z}$ (resp. $\frac{\partial f / \partial y}{\partial g / \partial y}>\frac{\partial f / \partial z}{\partial g / \partial z}$ ). Since increasing $y^{*}$ and decreasing $z^{*}$ (resp. increasing $z^{*}$ and decreasing $\left.y^{*}\right)$ cannot give a better solution for the problem, it must be the case that $y^{*}=1$ or $z^{*}=\beta / h$ (resp. $\left.y^{*}=0\right)$.

(iii) We have $\frac{\partial f}{\partial x}=0$ only if $x=h-1$, and $\frac{\partial f}{\partial x}<0$ only if $1 \leq x<h-1$. Therefore, if $x^{*}>h-1$, decreasing $x^{*}$ would give smaller objective value.

5.1. Covering Equilibria with $h \in\{3,4,6\}$

In this case we lower bound $\Omega_{1}$ by $(1+\beta) / 3$, which along with Observation 1 implies the upper bound of 3 on $R / M^{*}$ for $h \in\{3,4,6\}$.

LEMMA 5.7. For $h \in\{3,4,6\}, \Omega_{1} \geq \frac{1+\beta}{3}$.

PROOF. Let $\left(x^{*}, y^{*}, z^{*}\right)$ denote an optimal solution to problem (21). Notice that $x^{*} \in$ $[h-1]$ is an integer, which cannot be equal to the noninteger $\chi$ for any $h \in\{3,4,6\}$. By Lemma 5.6(i)-(ii), we have $z^{*}=\beta / h$ or $y^{*}=1$ if $x^{*}<\chi$ and $y^{*}=0$ otherwise. We calculate and estimate $\Omega_{1}=f\left(x^{*}, y^{*}, z^{*}\right)$ in Tables I and II below by checking all necessary $x^{*}$, using Lemma 5.6(iii) (see the fourth column of Table I and the third column of Table II). Table I presents the cases for $x^{*}<\chi$ and $z^{*}=\beta / h$, where $y^{*} \in[0,1]$ is determined by $g\left(x^{*}, y^{*}, z^{*}\right)=0$, and Table II presents the cases for $y^{*} \in\{0,1\}$. In all cases we obtain the claimed lower bound.

\subsection{Covering Equilibria with $h \geq 7$}

In this section, we assume $h \geq 7$. Our goal is to prove the optimal objective value $\Omega_{2}$ of problem (22) is at most $(1+\beta) / 3$ for all $h \geq 7$. Throughout Section 5.2 , we assume $\left(x^{*}, y^{*}, z^{*}\right)$ to be a fixed optimal solution to (22) such that $y^{*}$ is minimum. In particular, since $f(x, 1, z)=f(x+1,0, z)$ and $g(x, 1, z)=f(x+1,0, z)$ for all $x>0, z \in \mathbb{R}$, we can assume without loss of generality that $y^{*}<1$. In the following, we distinguish among three cases:

1) $x^{*}=1$ (Claim 5.8),

2) $x^{*}>1$ and $y^{*}=0$ (Claim 5.12), and

3) $x^{*}>1$ and $0<y^{*}<1$ (Claim 5.13).

Our basic tool is the KKT conditions which $\left(x^{*}, y^{*}, z^{*}\right)$ must satisfy. For notational convenience, we also express the constraints $x \geq 1, y \in[0,1], z \geq \beta / h$ as $g_{i}(x, y, z) \leq 0$, $i=1,2,3,4$, respectively, where $g_{1}(x, y, z)=-x+1, g_{2}(x, y, z)=y-1, g_{3}(x, y, z)=-y$ and $g_{4}(x, y, z)=-z-\beta / h$. By the KKT conditions on the minimization problem (22), 
Table I. $\Omega_{1} \geq \frac{1+\beta}{3}$ when $x^{*}<\chi$ and $z^{*}=\frac{\beta}{h}$ for $h=3,4,6$.

\begin{tabular}{c|cc||c||rc|l}
\hline$h$ & $z^{*}$ & $\chi$ & $x^{*}$ & $g\left(x^{*}, y^{*}, z^{*}\right)=0$ & $y^{*}$ & \multicolumn{1}{c}{$\Omega_{1}=f\left(x^{*}, y^{*}, z^{*}\right)$} \\
\hline 3 & $\frac{\beta}{3}$ & 1.5 & 1 & $\frac{7}{6} y^{*}+2 z^{*}-1-\beta=0$ & $\frac{6}{7}+\frac{2 \beta}{7}$ & $\frac{1+\beta}{3}$ \\
\hline & & & 1 & $y^{*}+2 z^{*}-\frac{5}{4}-\beta=0$ & $\notin[0,1]$ & infeasible \\
4 & $\frac{\beta}{4}$ & 2.19 & 2 & $\frac{2}{3} y^{*}+2 z^{*}-\frac{1}{4}-\beta=0$ & $\frac{3}{8}+\frac{3}{4} \beta$ & $\frac{75-18 \beta}{32}>\frac{1+\beta}{3}$ \\
\hline & & & 1 & $\frac{5}{6} y^{*}+2 z^{*}-\frac{3}{2}-\beta=0$ & $\notin[0,1]$ & infeasible \\
6 & $\frac{\beta}{6}$ & \multirow{2}{*}{3.59} & 2 & $\frac{1}{2} y^{*}+2 z^{*}-\frac{2}{3}-\beta=0$ & $\notin[0,1]$ & infeasible \\
& & & 3 & $\frac{5}{12} y^{*}+2 z^{*}-\frac{1}{6}-\beta=0$ & $\frac{2}{5}+\frac{8 \beta}{5}$ & $\frac{71-21 \beta}{30}>\frac{1+\beta}{3} *$ \\
\hline
\end{tabular}

Note: ${ }^{*} \beta \leq 3 / 8$.

Table II. $\Omega_{1} \geq \frac{1+\beta}{3}$ when $x^{*}<\chi$ and $y^{*}=1$ (resp. $x^{*}>\chi$ and $\left.y^{*}=0\right)$ for $h=3,4,6$.

\begin{tabular}{c|c||c||cc|l}
\hline$h$ & $\chi$ & $x^{*}$ & $y^{*}$ & $z^{*}$ & $\Omega_{1}=f\left(x^{*}, y^{*}, z^{*}\right)$ \\
\hline 3 & 1.5 & 1,2 & $2-x^{*}$ & $\frac{\beta}{2}-\frac{1}{12}$ & $\frac{9-2 \beta}{4} \geq \frac{1+\beta}{3} \dagger$ \\
\hline \multirow{3}{*}{4} & \multirow{3}{*}{2.19} & 1 & 1 & $\frac{\beta}{2}+\frac{1}{8}$ & $\frac{19-4 \beta}{8} \geq \frac{1+\beta}{3}$ \\
& & 2,3 & $3-x^{*}$ & $\frac{\beta}{2}-\frac{5}{24}$ & $\frac{55-12 \beta}{24}>\frac{1+\beta}{3} \ddagger$ \\
\hline & & 1 & 1 & $\frac{\beta}{2}+\frac{1}{3}$ & $\frac{6-\beta}{2}>\frac{1+\beta}{3}$ \\
6 & \multirow{3}{*}{3.59} & 2 & 1 & $\frac{\beta}{2}+\frac{1}{12}$ & $\frac{29-6 \beta}{12}>\frac{1+\beta}{3}$ \\
& & 3,4 & $4-x^{*}$ & $\frac{\beta}{2}-\frac{1}{8}$ & $\frac{55-12 \beta}{24}>\frac{1+\beta}{3} \S$ \\
& & 5 & 0 & $\frac{\beta}{2}-\frac{19}{60}$ & $\frac{47-10 \beta}{20}>\frac{1+\beta}{3}$ \\
\hline
\end{tabular}

Note: ${ }^{\dagger} \beta \geq 1 / 2 . \quad \ddagger \beta \geq 5 / 6 . \quad{ }^{\S} \beta \geq 3 / 8$.

there exist constant $\lambda$ and nonnegative constants $\mu_{i}(1 \leq i \leq 4)$ such that

$$
\begin{aligned}
& \nabla f\left(x^{*}, y^{*}, z^{*}\right)+\lambda \nabla g\left(x^{*}, y^{*}, z^{*}\right)+\sum_{i=1}^{4} \mu_{i} \nabla g_{i}\left(x^{*}, y^{*}, z^{*}\right)=\mathbf{0} \\
& \mu_{i} g_{i}\left(x^{*}, y^{*}, z^{*}\right)=0 \text { for } i \in[4] .
\end{aligned}
$$

Case 1. $x^{*}=1$. This case is handled by the following claim.

Claim 5.8. $\min \{f(x, y, z) \mid g(x, y, z)=0, x=1, y \in[0,1]\}>(1+\beta) / 3$.

Proof. If $x=1$, we have $z=1+\frac{\beta}{2}-\frac{3}{2 h}-\left(\frac{1}{h}+\frac{1}{4}\right) y$ from the constraint $g(x, y, z)=0$. It follows that $f(x, y, z)=h+\frac{5}{2 h}-\left(\frac{h}{2}-\frac{1}{4}-\frac{2}{h}\right) y-3+\frac{\beta}{2}$, which together with $h \geq 7$, $y \leq 1$ and $\beta \geq 0$ implies $f(x, y, z) \geq f(1,1, z)=\frac{h}{2}+\frac{9}{2 h}-\frac{11}{4}+\frac{\beta}{2}>\frac{7}{2}-\frac{11}{4}+\frac{\beta}{2}>\frac{1+\beta}{3}$.

Before turning to the next two cases, we prove a few technical lemmas.

Claim 5.9. If $h \geq 7$ and $1+\beta-\frac{1+2 \beta}{h}>\frac{\sqrt{2 h^{2}-h}}{h}-\frac{2}{\sqrt{2 h^{2}-h}}$, then $\beta>\sqrt{2}-1$.

Proof. The function $1+\beta-\frac{1+2 \beta}{h}$ increases in $\beta$ for $h>2$, and $\sqrt{2}-\frac{2 \sqrt{2}-1}{h} \leq$ $\frac{\sqrt{2 h^{2}-h}}{h}-\frac{2}{\sqrt{2 h^{2}-h}}$ for $h \geq 7$.

In the next two lemmas, we write $\beta_{-1}:=\beta-1, \beta_{1}:=\beta+1$ and $\beta_{2}:=2 \beta+1$ for brevity.

LeMmA 5.10. For any constant $\beta \in[0, \sqrt{2}-1)$, if variables $\hbar$ and $x$ satisfy $\hbar \geq 7$ and $x=\frac{1}{4}\left(\beta_{1} \hbar-\beta_{2}+\sqrt{\left(\beta_{1} \hbar-\beta_{2}\right)^{2}+8 \hbar}\right)$, then $\Psi(\hbar):=\frac{\hbar}{x}+\frac{x}{\hbar}-\frac{\beta}{\hbar} \geq \Psi(7)$. 
Proof. Notice that $\hbar=\frac{2 x^{2}+\beta_{2} x}{\beta_{1} x+1}$. Thus $\Psi(\hbar)$ can be considered as the function of $x$, which we write as $\psi(x)$.

$$
\psi(x)=\frac{2 x+\beta_{2}}{\beta_{1} x+1}+\frac{\beta_{1} x-\beta_{1} \beta+1}{2 x+\beta_{2}}-\frac{\beta}{2 x^{2}+\beta_{2} x}=\Psi(\hbar) .
$$

It is easy to check that $x$ is monotonically increasing in $\hbar$; in particular $\hbar \geq 7$ along with $\beta \geq 0$ implies $x \geq 3.897$.

Moreover, to prove the lemma, we only need to show that $\psi(x)$ is monotonically increasing in $x$. Observe that the last term in the derivative of the above expression

$$
\frac{d \psi}{d x}=\frac{1-3 \beta-2 \beta^{2}}{\left(\beta_{1} x+1\right)^{2}}+\frac{4 \beta^{2}+5 \beta-1}{\left(2 x+\beta_{2}\right)^{2}}+\frac{\beta\left(4 x+\beta_{2}\right)}{\left(2 x^{2}+\beta_{2} x\right)^{2}}
$$

is positive. It suffices to verify

$$
\frac{1-3 \beta-2 \beta^{2}}{\left(\beta_{1} x+1\right)^{2}}+\frac{4 \beta^{2}+5 \beta-1}{\left(2 x+\beta_{2}\right)^{2}} \geq 0 .
$$

In case of $0 \leq \beta \leq \frac{1}{4}(\sqrt{17}-3)<0.2808$, we obtain $1-3 \beta-2 \beta^{2} \geq \max \left\{0,1-5 \beta-4 \beta^{2}\right\}$, and (25) is true as $0<\frac{\left(\beta_{1} x+1\right)^{2}}{\left(2 x+\beta_{2}\right)^{2}} \leq 1$ for $0 \leq \beta \leq \sqrt{2}-1$.

In case of $\beta \in\left(\frac{1}{4}(\sqrt{17}-3), \sqrt{2}-1\right) \subset[0.28, \sqrt{2}-1)$, we have $2 \beta^{2}+3 \beta-1>0$. It can be seen from $x \geq 3.897$ that $(1.7 \beta-0.3) x>2 \beta-0.7$, implying $\frac{2 x+\beta_{2}}{\beta_{1} x+1} \leq 1.7$. On the other hand, (25) follows from

$$
\frac{4 \beta^{2}+5 \beta-1}{2 \beta^{2}+3 \beta-1}>2+\frac{1-(\sqrt{2}-1)}{2(\sqrt{2}-1)^{2}+3(\sqrt{2}-1)-1}=3>1.7^{2} \geq \frac{\left(2 x+\beta_{2}\right)^{2}}{\left(\beta_{1} x+1\right)^{2}} .
$$

The lemma is proved.

LEMMA 5.11. For any constant $\beta \in[0,0.13)$, if variables $\hbar$ and $x$ satisfy $\hbar=$ $\frac{(2 x+1)^{2}+\beta_{2}(2 x+1)+1}{\beta_{1}(2 x+1)+2} \geq 7$, then function $\Psi(x) \equiv \frac{\hbar}{x}+\frac{x}{\hbar}-\left(\frac{\hbar}{x(x+1)}-\frac{1}{\hbar}\right) \frac{x+1}{2 x+1}-\frac{\beta}{\hbar}>2.36$.

PROOF. Let $u=2 x+1$. Then $\hbar=\frac{u^{2}+\beta_{2} u+1}{\beta_{1} u+2} \geq 7$, and $u=\frac{\beta_{1} \hbar-\beta_{2}+\sqrt{\left(\beta_{1} \hbar-\beta_{2}\right)^{2}+8 \hbar-4}}{2}$ is lower bounded by $\frac{\hbar-1+\sqrt{(\hbar-1)^{2}+8 \hbar-4}}{2} \geq \frac{6+\sqrt{88}}{2}>7.69$, as $\hbar \geq 7$ and $\beta \geq 0$.

It is routine to check that $\Psi(x)=\frac{2 \hbar}{u}+\frac{u}{2 \hbar}+\frac{1}{2 \hbar u}-\frac{\beta}{\hbar}$, and it is a function $\psi$ of $u$ with derivative $d \psi / d u$ as follows:

$$
\begin{aligned}
\psi(u):= & \frac{\beta_{1} u^{3}+\left(2-2 \beta \beta_{1}\right) u^{2}+(1-3 \beta) u+2}{2 u\left(u^{2}+\beta_{2} u+1\right)}+\frac{2\left(u^{2}+\beta_{2} u+1\right)}{u\left(2+\beta_{1} u\right)}=\Psi(x) . \\
\frac{d \psi}{d u}= & \frac{\left(4 \beta_{1} \beta+\beta_{-1}\right) u^{4}+8 \beta u^{3}+\left(4 \beta_{-1} \beta_{1}-\beta_{1}\right) u^{2}-4 \beta_{2} u-2}{2 u^{2}\left(u^{2}+\beta_{2} u+1\right)^{2}} \\
& +\frac{2\left(1-3 \beta-2 \beta^{2}\right) u^{2}-4 \beta_{1} u-4}{u^{2}\left(2+\beta_{1} u\right)^{2}}
\end{aligned}
$$

By $u \geq 7.69$ and $\beta \in[0,0.13)$, it is easy to see that the numerator of the second term in the above expression of $d \psi / d u$ is positive. Since $\sqrt{2}\left(u^{2}+\beta_{2} u+1\right)>u\left(2+\beta_{1} u\right)$ holds for 
any $\beta \in[0,0.13)$, we have

$$
\begin{aligned}
\frac{d \psi}{d u}> & \frac{\left(4 \beta_{1} \beta+\beta_{-1}\right) u^{4}+8 \beta u^{3}+\left(4 \beta_{-1} \beta_{1}-\beta_{1}\right) u^{2}-4 \beta_{2} u-2}{2 u^{2}\left(u^{2}+\beta_{2} u+1\right)^{2}} \\
& +\frac{2\left(1-3 \beta-2 \beta^{2}\right) u^{4}-4 \beta_{1} u^{3}-4 u^{2}}{2 u^{2}\left(u^{2}+\beta_{2} u+1\right)^{2}} \\
= & \frac{(1-\beta) u^{4}-4(1-\beta) u^{3}-\left(9+\beta-4 \beta^{2}\right) u^{2}-4(2 \beta+1) u-2}{2 u^{2}\left(u^{2}+\beta_{2} u+1\right)^{2}} \\
\geq & \frac{0.87 u^{4}-4 u^{3}-9.0624 u^{2}-5.04 u-2}{2 u^{2}\left(u^{2}+\beta_{2} u+1\right)^{2}}
\end{aligned}
$$

The numerator is positive as $u>7.69$. Therefore $d \psi / d u>0$.

Using $u>7.69$ and $\beta<0.13$, we obtain $\Psi(x)=\psi(u) \geq \psi(7.69) \geq \frac{2 \hbar}{7.69}+\frac{7.69}{2 \hbar}+\frac{1}{15.38 \hbar}-$ $\frac{0.13}{\hbar}=\frac{200 \hbar}{769}+\frac{581367}{153800 \hbar}$, which increases in $\hbar$ for all $\hbar \geq 7$. Thus $\Psi(x) \geq \frac{200 \times 7}{769}+\frac{581367}{153800 \times 7}>$ 2.36. $\square$

Case 2. $x^{*}>1$ and $y^{*}=0$. We begin by considering the following relaxed problem, which does not have a bound on $z$.

$$
\Omega_{3}:=\min \{f(x, y, z) \mid g(x, y, z)=0, x \geq 1, y=0\} .
$$

Clearly $\Omega_{3} \leq \Omega_{2}$. The KKT conditions applied to (26) assert that $\Omega_{3}$ is attained at some feasible solution $(x, z)$ of (26) for which there exist constants $\theta$ and $\eta$ such that

$$
\begin{aligned}
\nabla f(x, 0, z)+\theta \nabla g(x, 0, z)+\eta \nabla(-x+1) & =\mathbf{0} \\
\eta(-x+1) & =0
\end{aligned}
$$

It follows that $\Omega_{3}$ is attained either when $x=1$ or when $x>1 \Rightarrow \eta=0 \Rightarrow \frac{\partial f / \partial x}{\partial g / \partial x}=$ $-\theta=\frac{\partial f / \partial z}{\partial g / \partial z}$ holds at $(x, 0, z)$. In the former case, we are done by Claim 5.8. In the latter case, Claim 5.5(iii) gives $x=\nu$, and therefore $g(x, 0, z)=g(\nu, 0, z)=0$ implies

$$
z=\left(\frac{h-1-2 \nu}{h}+\frac{1}{\nu}+\beta\right) / 2=: z_{2} .
$$

Notice that $\Omega_{3}=f\left(\nu, 0, z_{2}\right)=\frac{4 \sqrt{2 h^{2}-h}+1}{2 h}+\frac{\beta-5}{2}$ increases in $h$ for $h \geq 7$, and hence $\Omega_{3} \geq \frac{4 \sqrt{91}+1}{14}+\frac{\beta-5}{2}$, which is greater than $\frac{1+\beta}{3}$ if $\beta \geq \sqrt{2}-1$. This together with $\Omega_{3} \leq \Omega_{2}$ verifies the following

$$
\Omega_{2}>(1+\beta) / 3 \text { if } \beta \geq \sqrt{2}-1 .
$$

We next turn back to (22), and investigate its optimal solution $\left(x^{*}, 0, z^{*}\right)$.

Claim 5.12. If $x^{*}>1$ and $y^{*}=0$, then $\Omega_{2} \geq(1+\beta) / 3$.

Proof. If $z^{*}>\beta / h$, since $x^{*}>1$, the KKT conditions (23)-(24) imply that $\mu_{1}=$ $\mu_{4}=0$ and $\frac{\partial f / \partial x}{\partial g / \partial x}=-\lambda=\frac{\partial f / \partial z}{\partial g / \partial z}$ holds at $\left(x^{*}, 0, z^{*}\right)$. Using $y^{*}=0$ and Lemma 5.5(iii), we obtain $x^{*}=\nu$. In turn $g\left(x^{*}, 0, z^{*}\right)=0$ gives $z^{*}=z_{2}$. So $z_{2}>\beta / h$, which reads $1+\beta-\frac{1+2 \beta}{h}>\frac{\sqrt{2 h^{2}-h}}{h}-\frac{2}{\sqrt{2 h^{2}-h}}$. It follows from Lemma 5.9 that $\beta>\sqrt{2}-1$, and further from (27) that $\Omega_{2}>(1+\beta) / 3$.

If $z^{*}=\beta / h$, by (27), we only need to consider the case where $\beta \in[0, \sqrt{2}-1)$. The constraint $g\left(x^{*}, 0, z^{*}\right)=\frac{2 x^{*}}{h}-\frac{1}{x^{*}}+2 z^{*}-\frac{h-1}{h}-\beta$ gives

$$
x^{*}=\frac{1}{4}\left((\beta+1) h-(2 \beta+1)+\sqrt{((\beta+1) h-(2 \beta+1))^{2}+8 h}\right):=x^{*}(h) .
$$


It follows from Lemma 5.10 that $f\left(x^{*}, 0, z^{*}\right)=\frac{h}{x^{*}}+\frac{x^{*}}{h}-\frac{\beta}{h}-2+\beta \geq \frac{7}{x^{*}(7)}+\frac{x^{*}(7)}{7}+\frac{6 \beta}{7}-2$. This value is easily checked to be $\frac{15}{28} \sqrt{(6+5 \beta)^{2}+56}-\frac{41}{28} \beta-\frac{67}{14}$, which is smaller than $(1+\beta) / 3$ for $\beta \geq 0$.

Case 3. $x^{*}>1$ and $0<y^{*}<1$. In this case, the KKT conditions (23)-(24) imply $\mu_{i}=0$ for $1 \leq i \leq 3$ and $\frac{\partial f / \partial y}{\partial g / \partial y}=-\lambda=\frac{\partial f / \partial x}{\partial g / \partial x}$ holds at $\left(x^{*}, y^{*}, z^{*}\right)$. In turn, Lemma 5.5(i) asserts $y^{*}=\frac{x^{*}+1}{2 x^{*}+1}$, implying

$$
\Omega_{2} \geq \Omega_{4}:=\min \left\{f(x, y, z) \mid g(x, y, z)=0, x \geq 1, y=\frac{x+1}{2 x+1}\right\} .
$$

From the KKT conditions on the minimization (28), we deduce that $\Omega_{4}$ is attained at some feasible solution $(x, y, z)$ of (28) for which there exist constants $\theta_{1}, \theta_{2}, \eta_{1}, \eta_{2}$ such that

$$
\begin{aligned}
\nabla f(x, y, z)+\theta_{1} \nabla g(x, y, z)+\theta_{2} \nabla\left(y-\frac{x+1}{2 x+1}\right)+\eta \nabla(-x+1) & =\mathbf{0} \\
\eta(-x+1) & =0
\end{aligned}
$$

It follows that $\Omega_{4}$ is attained either when $x=1$ or when $x>1$, in which case $\eta=0$ by (30). In the former case, we are again done by Claim 5.8. In the latter case, from (29) we find

$$
\begin{aligned}
& \frac{\partial f}{\partial x}+\theta_{1} \frac{\partial g}{\partial x}=-\frac{\theta_{2}}{(2 x+1)^{2}} \\
& \frac{\partial f}{\partial y}+\theta_{1} \frac{\partial g}{\partial y}=-\theta_{2} \\
& \frac{\partial f}{\partial z}+\theta_{1} \frac{\partial g}{\partial z}=0
\end{aligned}
$$

Since $y=\frac{x+1}{2 x+1}$, Lemma 5.5(i) asserts $\frac{\partial f}{\partial x}=\frac{\partial f}{\partial y}$ and $\frac{\partial g}{\partial x}=\frac{\partial g}{\partial y}$, which along with (31) and (32) enforce $\theta_{2}=0$. In turn from (31) and (33) we derive $\frac{\partial f / \partial x}{\partial g / \partial x}=-\theta_{1}=\frac{\partial f / \partial z}{\partial g / \partial z}$. By $y=\frac{x+1}{2 x+1}$, Lemma 5.5(iv) enforces $x=\chi$. Hence from $g(x, y, z)=0$ we obtain

$$
z=\frac{1}{2}\left(1+\beta-\frac{1+2 \chi}{h}+\frac{1}{\chi}-\left(\frac{2}{h}+\frac{1}{\chi(\chi+1)}\right) \cdot \frac{\chi+1}{2 \chi+1}\right)=: z_{3}
$$

It follows that $\Omega_{4}=f\left(\chi, \frac{\chi+1}{2 \chi+1}, z_{3}\right)=\frac{4 \sqrt{2 h^{2}-h+1}+1}{2 h}+\frac{\beta-5}{2}$, which increases in $h$ for $h \geq 7$. So $\Omega_{4}$ is lower bounded by $\frac{4 \sqrt{92}+1}{14}+\frac{\beta-5}{2}$, which is greater than $\frac{1+\beta}{3}$ if $\beta>\frac{116-12 \sqrt{92}}{7}>$ 0.13 . Since $\Omega_{2} \geq \Omega_{4}$ in either case, we have shown that

$$
\Omega_{2}>(1+\beta) / 3 \text { if } \beta \geq 0.13 \text {. }
$$

Next we again focus on the optimal solution $\left(x^{*}, y^{*}, z^{*}\right)$ of (22).

Claim 5.13. If $x^{*}>1$ and $0<y^{*}<1$, then $\Omega_{2} \geq(1+\beta) / 3$.

Proof. If $z^{*}>\beta / h$, by $x^{*}>1$ and the KKT conditions (23)-(24), we obtain $\mu_{1}=$ $\mu_{4}=0$ and $\frac{\partial f / \partial x}{\partial g / \partial x}=-\lambda=\frac{\partial f / \partial z}{\partial g / \partial z}$ at $\left(x^{*}, y^{*}, z^{*}\right)$, which is equivalent to $x^{*}=\chi$ by $y^{*}=$ $\frac{x^{*}+1}{2 x^{*}+1}$ and Lemma 5.5(iv). In turn we have $z^{*}=z_{3}$ by using $g\left(\chi, \frac{\chi+1}{2 \chi+1}, z^{*}\right)=0$. Now $z_{3}>\beta / h$ reads $1+\beta-\frac{1+2 \beta}{h}>\frac{\sqrt{2 h^{2}-h+1}}{h}-\frac{2}{\sqrt{2 h^{2}-h+1}}+\frac{1}{h \sqrt{2 h^{2}-h+1}}$. The right-hand 
side of this inequality is larger than $\frac{\sqrt{2 h^{2}-h}}{h}-\frac{2}{\sqrt{2 h^{2}-h}}$. It follows from Claim 5.9 that $\beta>\sqrt{2}-1>0.13$, and further from (34) that $\Omega_{2}>(1+\beta) / 3$.

If $z^{*}=\beta / h$, by (34), it suffices to consider $\beta \in[0,0.13)$. From $g\left(x^{*}, y^{*}, z^{*}\right)=$ $g\left(x^{*}, \frac{x^{*}+1}{2 x^{*}+1}, \frac{\beta}{h}\right)=0$ we get

$$
h=\frac{\left(2 x^{*}+1\right)^{2}+(2 \beta+1)\left(2 x^{*}+1\right)+1}{(\beta+1)\left(2 x^{*}+1\right)+2} .
$$

Under this equation for $h \geq 7$ and $\beta \in[0,0.13)$, Lemma 5.11 asserts

$$
\Omega_{2}=f\left(x^{*}, y^{*}, z^{*}\right)=\frac{h}{x^{*}}+\frac{x^{*}}{h}-\left(\frac{h}{x^{*}\left(x^{*}+1\right)}-\frac{1}{h}\right) \frac{x^{*}+1}{2 x^{*}+1}-\frac{\beta}{h}-2+\beta>0.36+\beta,
$$

which is obviously greater than $\frac{1+\beta}{3}$.

To sum up, we have shown the following result.

LEMMA 5.14. If $\cup_{i \in[h]} \mathcal{N}_{i}=R$ and $h>2$, then $R / M^{*} \leq 3$.

6. THE CASES $H=1$ AND $H=2$

LEMMA 6.1. Let $\mathcal{I}$ be an SRR instance with $h=1$. Then $M / M^{*} \leq 2$.

Proof. Only the case $k=2$ is relevant. By assumption, we have $\mathcal{N}_{1}=\mathcal{R} \backslash \mathcal{Q}_{1}$ and we have $\mathcal{N}_{2}=\mathcal{Q}_{2}$. That is, we have $\pi^{*}=\left\{\mathcal{Q}_{1}, \mathcal{Q}_{2}\right\}$ and we have $\pi^{N}=\left\{\mathcal{N}_{1}, \mathcal{Q}_{2}\right\}$. In particular, we have $Q_{1}+\left\|\mathcal{Q}_{1}\right\|_{a}=Q_{1}^{*}$. Hence, $Q_{1}+\left\|\mathcal{Q}_{1}\right\|_{a} \leq M^{*}$. Since $\pi^{N}$ is a Nash equilibrium, it holds that $N_{1} \leq Q_{1}+\left\|\mathcal{Q}_{1}\right\|_{a} \leq M^{*}$. We thus get

$$
M \leq R=N_{1}+Q_{1} \leq 2 M^{*}
$$

as desired.

LEMMA 6.2. Let $\mathcal{I}$ be an $S R R$ instance with $h=2$ and $\cup_{i=1,2} \mathcal{N}_{i} \neq \mathcal{R}$. Then $M / M^{*} \leq 2$.

Proof. We first consider the case that $\mathcal{I}$ is nonsingular. Then $k=h=2$ by definition. Assume without loss of generality that $N_{1} \leq N_{2}$. Since $\pi^{N}$ is a Nash equilibrium, it holds that $N_{2} \leq Q_{2}+\left\|\mathcal{Q}_{2}\right\|_{a}$, which, by the fact that $k=h=2$, is at most $2 Q_{2}^{*}$. Therefore, $M=N_{2} \leq 2 Q_{2}^{*} \leq 2 M^{*}$.

Let us now consider the case that $\mathcal{I}$ is singular. That is, we have $k=3$ and $M=N_{3}$. First note that we can rewrite

$$
\mathcal{Q}_{1}=\mathcal{R} \backslash \mathcal{N}_{1}=\left(\mathcal{N}_{2} \cup \mathcal{Q}_{2}\right) \backslash \mathcal{N}_{1}=\left(\mathcal{N}_{2} \backslash \mathcal{N}_{1}\right) \cup\left(\mathcal{Q}_{2} \backslash \mathcal{N}_{1}\right)=\left(\mathcal{N}_{2} \backslash\left(\mathcal{N}_{1} \cap \mathcal{N}_{2}\right)\right) \cup\left(\mathcal{Q}_{1} \cap \mathcal{Q}_{2}\right)
$$

and, similarly, we have $\mathcal{Q}_{2}=\left(\mathcal{N}_{1} \backslash\left(\mathcal{N}_{1} \cap \mathcal{N}_{2}\right)\right) \cup\left(\mathcal{Q}_{1} \cap \mathcal{Q}_{2}\right)$. Using this and the fact that $\pi^{N}$ is a Nash routing, we obtain the following two inequalities.

$$
\begin{aligned}
& N_{1} \leq Q_{1}+\left\|\mathcal{Q}_{1}\right\|_{a}=N_{2}-\ell^{N}\left(\mathcal{N}_{1} \cap \mathcal{N}_{2}\right)+\ell^{N}\left(\mathcal{Q}_{1} \cap \mathcal{Q}_{2}\right)+\left\|\mathcal{Q}_{1}\right\|_{a} \\
& N_{2} \leq Q_{2}+\left\|\mathcal{Q}_{2}\right\|_{a}=N_{1}-\ell^{N}\left(\mathcal{N}_{1} \cap \mathcal{N}_{2}\right)+\ell^{N}\left(\mathcal{Q}_{1} \cap \mathcal{Q}_{2}\right)+\left\|\mathcal{Q}_{2}\right\|_{a}
\end{aligned}
$$

From this we get

$$
2 \ell^{N}\left(\mathcal{N}_{1} \cap \mathcal{N}_{2}\right) \leq 2 \ell^{N}\left(\mathcal{Q}_{1} \cap \mathcal{Q}_{2}\right)+\left\|\mathcal{Q}_{1}\right\|_{a}+\left\|\mathcal{Q}_{2}\right\|_{a} .
$$


Furthermore, when comparing $Q_{i}^{*}$ and $Q_{i}$ for $i=1,2$, we can ignore player 3 , because it contributes the same to both values. Hence

$$
\begin{aligned}
Q_{1}^{*} & =\ell^{*}\left(\mathcal{Q}_{1} \cap \mathcal{N}_{2}\right)+\ell^{*}\left(\mathcal{Q}_{1} \cap \mathcal{Q}_{2}\right) \\
& =\ell^{N}\left(\mathcal{Q}_{1} \cap \mathcal{N}_{2}\right)+\ell^{N}\left(\mathcal{Q}_{1} \cap \mathcal{Q}_{2}\right)+2\left\|\mathcal{Q}_{1} \cap \mathcal{Q}_{2}\right\|_{a} \\
& =Q_{1}+2\left\|\mathcal{Q}_{1} \cap \mathcal{Q}_{2}\right\|_{a},
\end{aligned}
$$

and, similarly, $Q_{2}^{*}=Q_{2}+2\left\|\mathcal{Q}_{1} \cap \mathcal{Q}_{2}\right\|_{a}$ holds. From this we conclude

$$
\begin{aligned}
M^{*} & \geq Q_{1}^{*}=Q_{1}+2\left\|\mathcal{Q}_{1} \cap \mathcal{Q}_{2}\right\|_{a} \\
& =\ell^{N}\left(\mathcal{Q}_{1} \cap \mathcal{Q}_{2}\right)+\ell^{N}\left(\mathcal{Q}_{1} \backslash \mathcal{Q}_{2}\right)+2\left\|\mathcal{Q}_{1} \cap \mathcal{Q}_{2}\right\|_{a} \\
M^{*} & \geq Q_{2}^{*}=Q_{2}+2\left\|\mathcal{Q}_{1} \cap \mathcal{Q}_{2}\right\|_{a} \\
& =\ell^{N}\left(\mathcal{Q}_{1} \cap \mathcal{Q}_{2}\right)+\ell^{N}\left(\mathcal{Q}_{2} \backslash \mathcal{Q}_{1}\right)+2\left\|\mathcal{Q}_{1} \cap \mathcal{Q}_{2}\right\|_{a}
\end{aligned}
$$

and $M^{*} \geq N_{3}^{*} \geq N_{3}-2\left\|\mathcal{N}_{3} \cap \mathcal{N}_{1} \cap \mathcal{N}_{2}\right\|_{a}$. Notice that $\ell^{N}\left(\mathcal{Q}_{1} \backslash \mathcal{Q}_{2}\right) \geq\left\|\mathcal{Q}_{1} \backslash \mathcal{Q}_{2}\right\|_{a}$, $\ell^{N}\left(\mathcal{Q}_{2} \backslash \mathcal{Q}_{1}\right) \geq\left\|\mathcal{Q}_{2} \backslash \mathcal{Q}_{1}\right\|_{a}$ and $2\left\|\mathcal{N}_{3} \cap \mathcal{N}_{1} \cap \mathcal{N}_{2}\right\|_{a} \leq \ell^{N}\left(\mathcal{N}_{1} \cap \mathcal{N}_{2}\right)$. We may thus conclude $M=N_{3}$ is upper bounded by

$$
\begin{array}{rlr} 
& M^{*}+2\left\|\mathcal{N}_{3} \cap \mathcal{N}_{1} \cap \mathcal{N}_{2}\right\|_{a} \\
\leq & M^{*}+\ell^{N}\left(\mathcal{N}_{1} \cap \mathcal{N}_{2}\right) \\
\leq & M^{*}+\ell^{N}\left(\mathcal{Q}_{1} \cap \mathcal{Q}_{2}\right)+\frac{1}{2}\left\|\mathcal{Q}_{1}\right\|_{a}+\frac{1}{2}\left\|\mathcal{Q}_{2}\right\|_{a} & \text { by (36) } \\
\leq & 2 M^{*}-\frac{1}{2} \ell^{N}\left(\mathcal{Q}_{1} \backslash \mathcal{Q}_{2}\right)-2\left\|\mathcal{Q}_{1} \cap \mathcal{Q}_{2}\right\|_{a}-\frac{1}{2} \ell^{N}\left(\mathcal{Q}_{2} \backslash \mathcal{Q}_{1}\right)+\frac{\left\|\mathcal{Q}_{1}\right\|_{a}+\left\|\mathcal{Q}_{2}\right\|_{a}}{2} \quad \text { by (37), (38) } \\
\leq & 2 M^{*}-\frac{1}{2}\left\|\mathcal{Q}_{1} \backslash \mathcal{Q}_{2}\right\|_{a}-2\left\|\mathcal{Q}_{1} \cap \mathcal{Q}_{2}\right\|_{a}-\frac{1}{2}\left\|\mathcal{Q}_{2} \backslash \mathcal{Q}_{1}\right\|_{a}+\frac{\left\|\mathcal{Q}_{1}\right\|_{a}+\left\|\mathcal{Q}_{2}\right\|_{a}}{2} \\
\leq & 2 M^{*} .
\end{array}
$$

The lemma is proved.

LEMMA 6.3. If $\cup_{i \in[h]} \mathcal{N}_{i}=\mathcal{R}$ and $h \leq 2$, then $R / M^{*} \leq 3$.

Proof. When $h=1$, Lemma 6.1 implies the conclusion. By Lemma 3.3, it remains to consider $k=h=2$ and $\mathcal{N}_{1} \cup \mathcal{N}_{2}=\mathcal{R}$. Suppose without loss of generality that $\ell^{N}\left(Q_{1}\right) \leq \ell^{N}\left(Q_{2}\right)$. Note that $\mathcal{Q}_{2} \subseteq \mathcal{N}_{1}$ and thus $\mathcal{N}_{1}=\mathcal{Q}_{2} \cup\left(\mathcal{N}_{1} \cap \mathcal{N}_{2}\right)$. This yields $\ell^{N}\left(\mathcal{Q}_{2}\right)+\ell^{N}\left(\mathcal{N}_{1} \cap \mathcal{N}_{2}\right)=N_{1} \leq Q_{1}+\left\|\mathcal{Q}_{1}\right\|_{a}$, where the latter inequality stems from the fact that player 1 does not want to deviate in $\pi^{N}$. Together with the assumption $Q_{1} \leq Q_{2}$ we thus have $\ell^{N}\left(\mathcal{N}_{1} \cap \mathcal{N}_{2}\right) \leq Q_{1}-Q_{2}+\left\|\mathcal{Q}_{1}\right\|_{a} \leq\left\|\mathcal{Q}_{1}\right\|_{a} \leq M^{*}$. It follows from $\mathcal{N}_{i}=\mathcal{R} \backslash \mathcal{Q}_{i}, i=1,2$ that

$$
R=Q_{2}+Q_{1}+\ell^{N}\left(\mathcal{N}_{1} \cap \mathcal{N}_{2}\right) \leq Q_{2}+Q_{1}+M^{*} .
$$

Since $Q_{i}=Q_{i}^{*}$ for $i=1,2$, we obtain $R \leq Q_{1}^{*}+Q_{2}^{*}+M^{*} \leq 3 M^{*}$ as desired.

7. COVERING EQUILIBRIA WITH $H=5$

For the special case of $h=5$, we upper bound $R / M^{*}$ directly by using structural properties of the Nash equilibrium.

LEMMA 7.1. If $\cup_{i \in[h]} \mathcal{N}_{i}=\mathcal{R}$ and $h=5$, then $\frac{R}{M^{*}} \leq 3$.

Proof. Again, by Lemma 3.3, we only need to consider the case where $\pi^{N}$ is nonsingular. If $\pi^{N}(e) \geq 2$ for all $e \in E$, then $A_{1}=B_{1}=0$ in (5) and (7). Collecting terms in (5) gives $A_{3}+11 A_{4}+25 A_{5}+B_{3}+3 B_{4}+5 B_{5} \leq 5 A_{2}+B_{2}$, which is equivalent to 


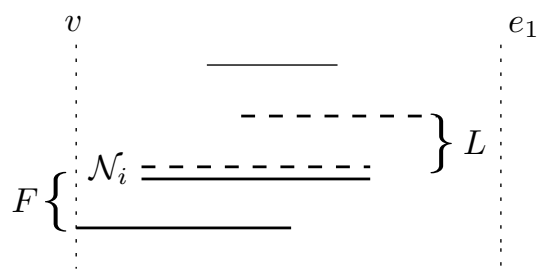

(i) $i \in F \cap L \neq \emptyset$

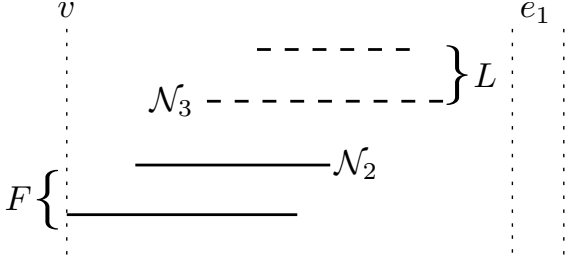

(ii) $2 \in F, 3 \in L$

Fig. 3. Splitting $\mathcal{R}$ at node $v$, where the Nash path $\mathcal{N}_{1}$ containing $e_{1}$ is not depicted.

$5\left(\sum_{i=2}^{5} i A_{i}+B_{i}\right) \leq 25 A_{2}+12 A_{3}-13 A_{4}-50 A_{5}+8 B_{2}+2 B_{3}-4 B_{4}-10 B_{5}$. It follows from (7) that

$$
\begin{aligned}
\frac{R}{M^{*}} & \leq \frac{5\left(\sum_{i=2}^{5} i A_{i}+B_{i}\right)}{\sum_{i=2}^{5}\left((5-i)^{2} A_{i}+(5-i) B_{i}\right)} \\
& \leq \frac{25 A_{2}+12 A_{3}+8 B_{2}+2 B_{3}}{9 A_{2}+4 A_{3}+A_{4}+4 B_{1}+3 B_{2}+2 B_{3}+B_{4}} \\
& \leq 3 .
\end{aligned}
$$

Therefore, we may assume without loss of generality that there exists a link $e_{1} \in \mathcal{N}_{1}$ with $\pi^{N}\left(e_{1}\right)=1$. Note that this implies $e_{1} \notin \cup_{i=2}^{5} \mathcal{N}_{i}$ and, thus, $\cup_{i=2}^{5} \mathcal{N}_{i} \neq \mathcal{R}$. Starting from link $e_{1}$, let $v$ be the clockwise first node where the Nash path $\mathcal{N}_{i}$ of another player $i \in\{2,3,4,5\}$ starts. For the analysis, let us temporarily split the ring at node $v$, and put the nodes on a line from left to right, starting and ending with $v$. Then for each player in $\{2,3,4,5\}$, its Nash path is one line segment by $\cup_{i=2}^{5} \mathcal{N}_{i} \neq \mathcal{R}$ and definition of $v$. See Figure 3 for an illustration.

Let $F \subseteq\{2,3,4,5\}$ consist of two players with the leftmost left endpoints, and $L \subseteq$ $\{2,3,4,5\}$ consist of two agents with the rightmost right endpoints. (Going from left to right, $F$ are two of the first players that start, and $L$ are two of the last players that finish their Nash paths.)

If there exists a player $i$ that is in both $F$ and $L$-formally if $i \in F \cap L \neq \emptyset$ (see Figure 3(i) for an illustration), then the definitions of $F$ and $L$ guarantee that both to the left and to the right of the path $\mathcal{N}_{i}$ of $i$ in $\pi^{N}$, any link can only be used (in $\pi^{N}$ ) by at most one player $j \in\{2,3,4,5\}$ and possibly by the first player. It follows that $\pi^{N}(e) \leq 2$ and hence $3 \leq \pi^{*}(e)$ for all $e \in \mathcal{Q}_{i}$. In particular we have $\pi^{N}(e)+1 \leq \pi^{*}(e)$ for all $e \in \mathcal{Q}_{i}$. Since $\pi^{N}$ is a Nash equilibrium, we conclude that $N_{i} \leq Q_{i}+\left\|\mathcal{Q}_{i}\right\|_{a} \leq Q_{i}^{*} \leq M^{*}$, giving $R=N_{i}+Q_{i} \leq 2 M^{*}$ as desired.

Therefore, let us consider the case $F \cap L=\emptyset$. Without loss of generality, let player 2 be a player in $F$ with the rightmost right endpoint, and let player 3 be a player in $L$ with the leftmost left endpoint (an illustration is given by Figure 3(ii)). Then in $\pi^{N}$, any link to the right of $\mathcal{N}_{2}$ can only be used by players in $L \cup\{1\}$, i.e., it can be used by at most three players, and any link to the left of $\mathcal{N}_{2}$ can only be used by players in $(F \backslash\{2\}) \cup\{1\}$, i.e., it can be used by at most two players. Thus $\pi^{N}(e) \leq 3$ for every $e \in \mathcal{Q}_{2}$. Analogously we have $\pi^{N}(e) \leq 3$ for every $e \in Q_{3}$. Moreover, we see that $\left\{e \in \mathcal{Q}_{2} \mid \pi^{N}(e)=3\right\} \subseteq \mathcal{N}_{3} \cap \mathcal{N}_{1}$ and $\left\{e \in \bar{Q}_{3} \mid \pi^{N}(e)=3\right\} \subseteq \mathcal{N}_{2} \cap \mathcal{N}_{1}$. On the other hand, from the selections of player 2 from $F$, and player 3 from $L$, it is easy to see that $\left\{e \in \mathcal{Q}_{1} \mid \pi^{N}(e) \geq 3\right\} \subseteq \mathcal{N}_{2} \cap \mathcal{N}_{3}$. This implies the following useful inequality, valid for all assignments $\{r, s, t\}=\{1,2,3\}$ :

$$
\ell^{N}\left(\mathcal{Q}_{r} \backslash\left(\mathcal{N}_{s} \cap \mathcal{N}_{t}\right)\right)+\left\|\mathcal{Q}_{r} \backslash\left(\mathcal{N}_{s} \cap \mathcal{N}_{t}\right)\right\|_{a} \leq \ell^{*}\left(Q_{r} \backslash\left(\mathcal{N}_{s} \cap \mathcal{N}_{t}\right)\right) .
$$


Let $S=\{(1,2,3),(2,3,1),(3,1,2)\}$. Adding the Nash inequalities for the paths $\mathcal{N}_{i}$, $i=1,2,3$, and their alternatives gives

$$
\begin{aligned}
\sum_{i=1}^{3} N_{i} \leq & \sum_{i=1}^{3}\left(Q_{i}+\left\|\mathcal{Q}_{i}\right\|_{a}\right) \\
= & \sum_{(r, s, t) \in S}\left[\ell^{N}\left(\mathcal{Q}_{r} \cap \mathcal{N}_{s} \cap \mathcal{N}_{t}\right)+\ell^{N}\left(\mathcal{Q}_{r} \backslash\left(\mathcal{N}_{s} \cap \mathcal{N}_{t}\right)\right)\right] \\
& +\sum_{(r, s, t) \in S}\left[\left\|\mathcal{Q}_{r} \cap \mathcal{N}_{s} \cap \mathcal{N}_{t}\right\|_{a}+\left\|\mathcal{Q}_{r} \backslash\left(\mathcal{N}_{s} \cap \mathcal{N}_{t}\right)\right\|_{a}\right]
\end{aligned}
$$

It follows from (39) and $\left\|\mathcal{Q}_{1} \cap \mathcal{N}_{2} \cap \mathcal{N}_{3}\right\|_{a} \leq \ell^{*}\left(\mathcal{Q}_{1} \cap \mathcal{N}_{2} \cap \mathcal{N}_{3}\right)$ that

$$
\begin{aligned}
& \sum_{i=1}^{3} N_{i} \\
\leq & \sum_{(r, s, t) \in S}\left(\ell^{N}\left(\mathcal{Q}_{r} \cap \mathcal{N}_{s} \cap \mathcal{N}_{t}\right)+\ell^{*}\left(\mathcal{Q}_{r} \cap \mathcal{N}_{s} \cap \mathcal{N}_{t}\right)+\ell^{*}\left(\mathcal{Q}_{r} \backslash\left(\mathcal{N}_{s} \cap \mathcal{N}_{t}\right)\right)\right) \\
= & \sum_{(r, s, t) \in S} \ell^{N}\left(\mathcal{Q}_{r} \cap \mathcal{N}_{s} \cap \mathcal{N}_{t}\right)+\sum_{i=1}^{3} Q_{i}^{*} \\
= & \sum_{(r, s, t) \in S}\left(\ell^{N}\left(\mathcal{N}_{s} \cap \mathcal{N}_{t}\right)-\ell^{N}\left(\mathcal{N}_{r} \cap \mathcal{N}_{s} \cap \mathcal{N}_{t}\right)\right)+\sum_{i=1}^{3} Q_{i}^{*} \\
= & \sum_{1 \leq i<j \leq 3} \ell^{N}\left(\mathcal{N}_{i} \cap \mathcal{N}_{j}\right)-3 \ell^{N}\left(\mathcal{N}_{1} \cap \mathcal{N}_{2} \cap \mathcal{N}_{3}\right)+\sum_{i=1}^{3} Q_{i}^{*}
\end{aligned}
$$

thus implying

$$
\sum_{i=1}^{3} N_{i}-\sum_{1 \leq i<j \leq 3} \ell^{N}\left(\mathcal{N}_{i} \cap \mathcal{N}_{j}\right)+\ell^{N}\left(\mathcal{N}_{1} \cap \mathcal{N}_{2} \cap \mathcal{N}_{3}\right) \leq \sum_{i=1}^{3} Q_{i}^{*}
$$

Notice that the left-hand side of the above inequality equals $R$ and the right-hand side is at most $3 M^{*}$. The result follows.

\section{ADDITIONAL REMARKS}

\subsection{Lower Bounds for Polynomial Latencies}

Replace the latency functions in Figure 1 by $x^{d}$ for the top and bottom links, and $(1-$ $\left.2^{-d}\right) x^{d-1}=x^{d-1}-\frac{1}{2}\left(\frac{x}{2}\right)^{d}$ for the right and left links. Then the optimal cost is still 1 and the cost of the Nash equilibrium is $2 \cdot\left(2^{d-1}-\frac{1}{2}\right)+1=2^{d}$. This is indeed a Nash equilibrium, because for agent $i=1,2$, deviating would give a cost of $2^{d}$ as well.

\subsection{Complement Games}

We can extend our proof almost entirely to the more general class of games where each player can choose between a set of resources and its complement. For the main line of the proof, we have assumed that the ring was covered (Section 5). In the context of complement games, this means that every item (resource) was selected by at least one player. However, the only place where we have used this is in the proof of Lemma 5.1. Without the covering assumption, the bound $M \leq 2 R / 3$ increases to $M \leq 2 R / 3+$ 
Table III. $(1+\beta) / \Omega_{1} \leq 3.125$ for $h=5$.

\begin{tabular}{c|c|c|c|c|c}
\hline$x^{*}$ vs. $\chi=2.89 \ldots$ & $x^{*}$ & $y^{*} \in[0,1]$ & $z^{*} \in\left[\frac{\beta}{5},+\infty\right)$ & $\Omega_{1}=f\left(x^{*}, y^{*}, z^{*}\right)$ & $(1+\beta) / \Omega_{1}$ \\
\hline \multirow{3}{*}{$x^{*}<\chi$} & 1 & $\frac{3 \beta}{2}+\frac{14}{9}$ & $\frac{\beta}{5}$ & infeasible & infeasible $^{b}$ \\
& 1 & 1 & $\frac{\beta}{2}+\frac{1}{4}$ & $\frac{\beta}{2}+\frac{13}{20}$ & $<3$ \\
\cline { 2 - 6 } & 2 & $\frac{18 \beta}{17}+\frac{15}{17}$ & $\frac{\beta}{5}$ & $\frac{11 \beta}{85}+\frac{29}{85}$ & $\leq 3.125^{\natural}$ \\
& 1 & $\frac{\beta}{2}-\frac{1}{30}$ & $\frac{\beta}{2}+\frac{3}{10}$ & $\leq 3.125^{\sharp}$ \\
\hline \multirow{3}{*}{$x^{*}>\chi$} & 3 & 0 & $\frac{\beta}{2}-\frac{1}{30}$ & $\frac{\beta}{2}+\frac{3}{10}$ & $\leq 3.125^{\sharp}$ \\
\cline { 2 - 6 } & 4 & 0 & $\frac{\beta}{2}-\frac{11}{40}$ & $\frac{\beta}{2}+\frac{13}{40}$ & $<3 \|$ \\
\hline
\end{tabular}

Note: ${ }^{b} y^{*}>1 . \quad{ }^{\natural} \beta \leq 1 / 9 . \quad{ }^{\sharp} \beta \geq 1 / 9 . \quad \|_{\beta} \geq 11 / 12$.

$M^{*} /(3 h)$. This follows from inequality (4). Combining this with $R \leq 3 M^{*}$ we get $M \leq$ $(2+1 /(3 h)) M^{*}$.

This proves a price of anarchy of $2+1 /(3 h)$ for complement games with $h \neq 5$. In fact, for $h \in\{1,2\}$, Lemmas $6.1-6.3$ hold, giving $P o A \leq 2$ in the case. For $h=5$, we have $R \leq 3.125 M^{*}$ by similar analysis to that in Section 5.1 for $h \in\{3,4,6\}$. The details are given in Table III. From the above general bound $M \leq 2 R / 3+M^{*} /(3 h)$ it thus follows that for $h=5$ we have $M \leq(6.25 / 3+1 / 15) M^{*}=2.15 M^{*}$.

Summarizing all the above, we have shown that the PoA for complement games is at most 2.15. We conjecture that the exact value of the PoA is 2 .

\section{CONCLUDING REMARKS}

We have shown that the PoA of the network congestion game is two, when the network is a ring and the link latencies are linear. It is left open whether the PoA is exactly $2^{d}$ for polynomial latency functions of degree $d$. Another challenging open question is to analyze what happens in more complicated network topologies.

\section{ACKNOWLEDGMENTS}

This work would not have been started without Xujin Chen and Benjamin Doerr having been invited to the Sino-German Frontiers of Science Symposium (Qingdao, 2010) organized by the Humboldt Foundation. A follow-up visit of Xujin Chen at the MPI in 2010 was also funded by the Humboldt Foundation via a CONNECT grant. We thank the Humboldt Foundation for providing both means of support.

This work was also supported in part by NNSF of China under Grant No. 11222109, 11021161 and 10928102, by 973 Project of China under Grant No. 2011CB80800, by CAS under Grant No. kjcx-yw-s7, by CAS Program for Cross \& Cooperative Team of Science \& Technology Innovation.

Carola Doerr gratefully acknowledges support from a Google Europe Fellowship in Randomized Algorithms, a Feodor Lynen postdoctoral research fellowship of the Alexander von Humboldt Foundation, and from the Agence Nationale de la Recherche, project ANR-09-JCJC-0067-01.

Rob van Stee would like to thank Éva Tardos for interesting discussions.

\section{REFERENCES}

Aland, S., Dumrauf, D., Gairing, M., Monien, B., and Schoppmann, F. 2006. Exact price of anarchy for polynomial congestion games. In Proceedings of the 23rd Conference on Theoretical Aspects of Computer Science (STACS'06). STACS’06. Springer-Verlag, Berlin, Heidelberg, 218-229.

Anshelevich, E., Dasgupta, A., Kleinberg, J. M., Tardos, É., Wexler, T., and Roughgarden, T. 2004. The price of stability for network design with fair cost allocation. In Proceedings of the 45th Annual IEEE Symposium on Foundations of Computer Science (FOCS'04). FOCS '04. IEEE Computer Society, Washington, DC, USA, 295-304.

Anshelevich, E. and Zhang, L. 2008. Path decomposition under a new cost measure with applications to optical network design. ACM Trans. Algorithms 4, 1, Article 15, 20 pages. 
Awerbuch, B., Azar, Y., and Epstein, A. 2005. The price of routing unsplittable flow. In Proceedings of the 37th ACM symposium on Theory of Computing (STOC'05). STOC '05. ACM, New York, NY, USA, 57-66.

Blum, A., Kalai, A., and Kleinberg, J. M. 2001. Admission control to minimize rejections. In Proceedings of the 7th International Workshop on Algorithms and Data Structures (WADS'01), F. Dehne, J.-R. Sack, and R. Tamassia, Eds. Lecture Notes in Computer Science. Springer, 155-164.

Chen, B., Chen, X., and Hu, J. 2013. Stability vs. optimality in selfish ring routing. Acta Math. Sin. (Engl. Ser.), to appear.

Chen, B., Chen, X., and Hu, X. 2010. The price of atomic selfish ring routing. J. Comb. Optim. 19, 3, 258-278.

Cheng, C. T. 2004. Improved approximation algorithms for the demand routing and slotting problem with unit demands on rings. SIAM J. Discrete Math. 17, 3, 384-402.

Christodoulou, G. and Koutsoupias, E. 2005. The price of anarchy of finite congestion games. In Proceedings of the 37th ACM Symposium on Theory of Computing (STOC'05). STOC '05. ACM, New York, NY, USA, 67-73.

Czumaj, A. 2004. Selfish routing on the internet. In Handbook of Scheduling: Algorithms, Models, and Performance Analysis, J. Y. Leung, Ed. CRC Press, Chapter 42.

Gairing, M., Lücking, T., Mavronicolas, M., and Monien, B. 2006. The price of anarchy for restricted parallel links. Parallel Process. Lett. 16, 1, 117-132.

GLORIAD. 2013. Global ring network for advanced applications development. http://www.gloriad.org.

Koutsoupias, E. and Papadimitriou, C. H. 1999. Worst-case equilibria. In Proceedings of the 16th annual conference on Theoretical aspects of computer science (STACS'99). STACS '99. Springer, 404-413.

Koutsoupias, E. and Papadimitriou, C. H. 2009. Worst-case equilibria. Comput. Sci. Rev. 3, 2, 65 - 69.

Lin, H., Roughgarden, T., Tardos, É., and Walkover, A. 2011. Stronger bounds on braess's paradox and the maximum latency of selfish routing. SIAM J. Discrete Math. 25, 4, 1667-1686.

Mavronicolas, M. and Spirakis, P. G. 2001. The price of selfish routing. In Proceedings of the 33rd ACM Symposium on Theory of Computing (STOC'01). STOC '01. ACM, New York, NY, USA, 510-519.

Rosenthal, R. W. 1973. A class of games possessing pure-strategy nash equilibira. Internat. J. Game Theory $2,1,65-67$.

Roughgarden, T. 2003. The price of anarchy is independent of the network topology. J. Comput. System Sci. 67, 2, 341-364.

Roughgarden, T. and Tardos, É. 2002. How bad is selfish routing? J. ACM 49, 2, 236-259.

Schrijver, A., Seymour, P. D., and Winkler, P. 1998. The ring loading problem. SIAM J. Discrete Math. 11, 1, $1-14$.

Wang, B.-F. 2005. Linear time algorithms for the ring loading problem with demand splitting. J. Algorithms 54, 1, 45-57.

Received March 2013; revised July 2013; accepted September 2013 